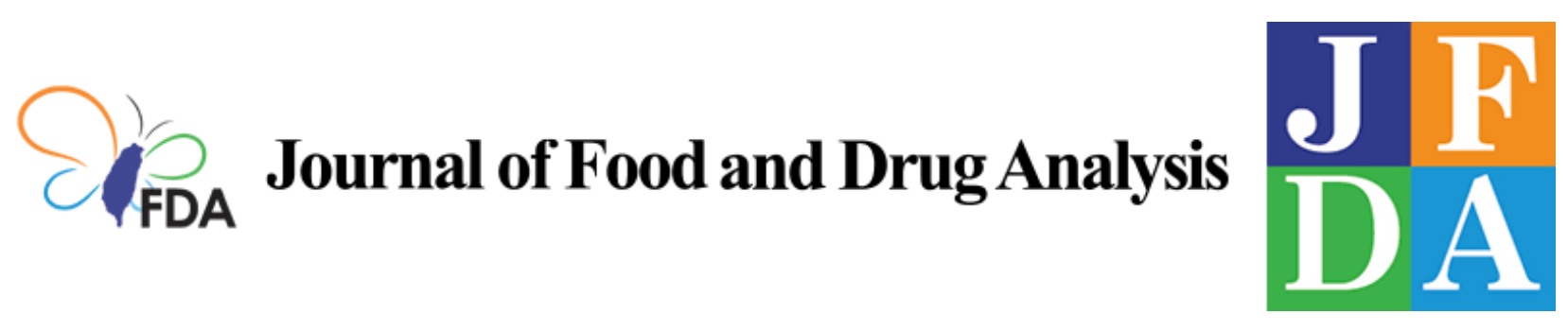

Volume 28 | Issue 4

Article 7

2020

\title{
Nanomaterial-based fluorescent biosensor for veterinary drug detection in foods
}

Follow this and additional works at: https://www.jfda-online.com/journal

Part of the Food Science Commons, Medicinal Chemistry and Pharmaceutics Commons, Pharmacology Commons, and the Toxicology Commons

(c) (i) (8)

This work is licensed under a Creative Commons Attribution-Noncommercial-No Derivative Works 4.0 License.

\section{Recommended Citation}

Tao, Xiaoqi; Peng, Yuanyuan; and Liu, Juewen (2020) "Nanomaterial-based fluorescent biosensor for veterinary drug detection in foods," Journal of Food and Drug Analysis: Vol. 28 : Iss. 4 , Article 7. Available at: https://doi.org/10.38212/2224-6614.1267

This Review Article is brought to you for free and open access by Journal of Food and Drug Analysis. It has been accepted for inclusion in Journal of Food and Drug Analysis by an authorized editor of Journal of Food and Drug Analysis. 


\title{
Nanomaterial-based fluorescent biosensors for veterinary drug detection in foods
}

\author{
Xiaoqi Tao ${ }^{\mathrm{a}, \mathrm{b}, *, 1}$, Yuanyuan Peng ${ }^{\mathrm{a}, 1}$, Juewen Liu ${ }^{\mathrm{b}, * *}$ \\ ${ }^{\text {a }}$ College of Food Science, Southwest University, Chongqing, 400715, China \\ ${ }^{\mathrm{b}}$ Department of Chemistry, Waterloo Institute for Nanotechnology, University of Waterloo, Waterloo, ON, N2L 3G1, Canada
}

\begin{abstract}
Veterinary drugs have been widely used in the food industry. Their residues in food products need to be tightly regulated to ensure food safety. In particular, some veterinary drugs are still used illegally, although they have already been banned. Nanomaterials are playing an increasingly important role in analytical chemistry due to their unique properties. Compared to traditional organic dyes and colloidal gold nanoparticle labels, fluorescent nanomaterials appear particularly attractive for the detection of veterinary drug residues. This review summarizes recent advancements of fluorescent biosensors using nanomaterials for the detection of veterinary drug residues in foods. The useful properties of each type of fluorescent nanomaterial are first discussed such as large Stokes shifts, long emission lifetime, and high quantum yields, which are useful for detection in food-related sample matrix. The following target recognition molecules are then reviewed individually including antibodies, aptamers, molecularly imprinted polymers, and metal ion coordination based ligands. Representative bioconjugation and assay methods are discussed for each recognition mechanism. Finally, a few future research directions are outlined in the last section of this review.
\end{abstract}

Keywords: Antibodies, Aptamers, Biosensors, Fluorescence, Nanoparticles

\section{Introduction}

$\mathrm{F}$ ood contamination is a major threat to human health, and recall of spoiled food can lead to huge economic losses in the food industry [1]. For various reasons, many illicit components exist in food products. Some are intentionally added, such as melamine for artificially boosting the nitrogen content that is used to reflect the protein content. Others are produced by food spoilage, or inadvertent exposure to trace toxicants, which may also increase the potential of food contamination [2].

Veterinary drug residues exist mainly in animalderived products such as meat, milk, and eggs. To ensure effective feeding and fast growth of animals, and prevention and treatment of animal diseases, various veterinary drugs were used, such as hormones, growth promoters, and antibiotics [3].
Therefore, potential drug residues in animalderived foods may cause adverse health effects such as cancer, disrupted hormone functions, and drug resistance [4,5]. For example, chloramphenicol can cause leukemia [6]; penicillin may cause the rash outbreaks [3]; while sulfanilamide and tetracycline are known for allergic reactions in susceptible bodies $[7,8]$. Therefore, the detection of veterinary drugs is of critical importance.

Technologies for detecting veterinary drug residues in foods are an important defense line for regulating market behavior and ensuring food safety. Detection of veterinary drugs is mainly needed by market regulation in animal farms, food manufacturing facilities, restaurants, retail markets, and wholesale markets [3], and it plays a crucial role in establishing public scientific health guidelines [9].

Many conventional methods were established for the detection of food contaminants. These methods

Received 11 May 2020; Received in revised form 17 July 2020; accepted 30 July 2020.

Available online 1 December 2020

* Corresponding author at: College of Food Science, Southwest University, Chongqing, 400715, China.

** Corresponding author at: Department of Chemistry, Waterloo Institute for Nanotechnology, University of Waterloo, Waterloo, ON, N2L 3G1, Canada. Fax: +1 5197460435

E-mail addresses: taoxiaoqi@swu.edu.cn (X. Tao), liujw@uwaterloo.ca (J. Liu).

1 These authors contributed equally to this work. 
include thin-layer chromatography (TLC) [10], highperformance liquid chromatography (HPLC) [11], liquid chromatography-mass spectrometry (LC-MS) [12], gas chromatography combined with mass spectrometric (GC-MS) [13], time-of-flight mass spectrometry (TOF-MS) [14] and capillary electrophoresis (CE) [15]. Nevertheless, the above methods have some inherent drawbacks, such as timeconsuming and needing expensive instruments $[9,16]$. These complicated and laborious methods were difficult to meet the needs of fast and on-site screening of massive samples.

Therefore, rapid, portable, and effective detection methods are critical to ensure food safety. The complexity of food sample matrix and difficulties associated with tracking food contaminants pose additional challenges. Over the past decade, a large number of biosensors were reported as supplementary detection tools offering exciting alternatives to traditional methods for food contaminant detection [17]. Antibodies and aptamers are the main sensing molecules to develop biosensors.

In recent years, nanomaterials have gained popularity in biosensors and become a key component in the detection of microbes [18], environmental pollutants [19], toxins [20], pesticides [21], and veterinary drug residues [9], because of their excellent optical, magnetic and adsorption properties [22]. The published reviews on nanomaterial-based biosensors for veterinary drug detection mainly focus on optical and electrochemical detection of antibiotics [16] or electrochemical and electrochemiluminescent sensors [4,9].

Optical biosensors have a few advantages owing to their simplicity, specificity, and sensitivity. Fluorescence biosensors as the most exploited for food contaminants detection, due to its high sensitivity, wide dynamic range and versatile detection methods [22]. A diverse range of nanomaterialbased fluorophores are often brighter (higher fluorescence quantum yield), more stable, and sometimes have sharper emission peaks compared to organic fluorophores, allowing highly sensitive and multiplexed detection [23]. In addition, nanomaterials were used as immobilization platforms due to their unique features, such as a high specific surface area, and surfaces that can be readily modified with ligands. This review focuses on fluorescent biosensors based on nanomaterials for detecting veterinary drugs. We aim to critically review the main sensor design ideas by considering the food sample matrix.

\section{Monitoring of veterinary drugs}

\subsection{Characteristics of veterinary drug}

Veterinary drugs can be divided into the following types: antibiotics, synthetic antibacterials, antiparasitic drugs, and growth-promoting agents [24]. The chemical structures of some representative veterinary drugs are illustrated in Fig. 1. Although acute poisoning caused by veterinary drug residues in foods is extremely unlikely, low-level and long-term uptake still can produce various chronic and accumulative toxicity [25]. The harm of intake of veterinary drug residues is often unnoticed until a substantial and irreversible symptom is produced. Thus, stringent maximum residue limits (MRL) for veterinary drugs in animal-derived foods have been established by various regulatory authorities, including the European Union (EU) [26], China [27], USA [28], and Japan [29]. For instance, the MRL of chloramphenicol considered by China and the EU in milk is $0.3 \mu \mathrm{g} \mathrm{L}^{-1}[26,27]$.

\subsection{Characteristics of veterinary drug residues}

Veterinary drug residues refer to the drug primitives, metabolites and impurities stored in cells, tissues or organs after giving the drugs to animals [30]. The use of banned and unqualified veterinary drugs, change of administration route, and non-compliance with the withdrawal period would cause veterinary drug residues [31]. The detection of veterinary drug residues is challenging for the following reasons. (1) Sensor signals have to be strong enough and the binding of the target has to be sufficiently specific when applied in a food sample matrix such as meat, milk, and eggs. (2) The detection needs to be sensitive enough to meet tight regulatory guidelines because the veterinary drug residues are generally at the level of part per billion ( $\mathrm{ppb})$.

\subsection{Food sample pretreatment}

As one of the key steps in veterinary drug residue analysis, food sample pretreatment directly affects the efficiency and accuracy of detection, and complicated pretreatment steps may increase the detection time [32]. Sample pretreatment starts from sampling to chemical analysis, including sample homogenization, reduction, sieving, centrifugation, filtration, antiseptic, and inhibition of degradation [33]. The complexity of food matrices frequently troubles analysis. In designing sample treatment 


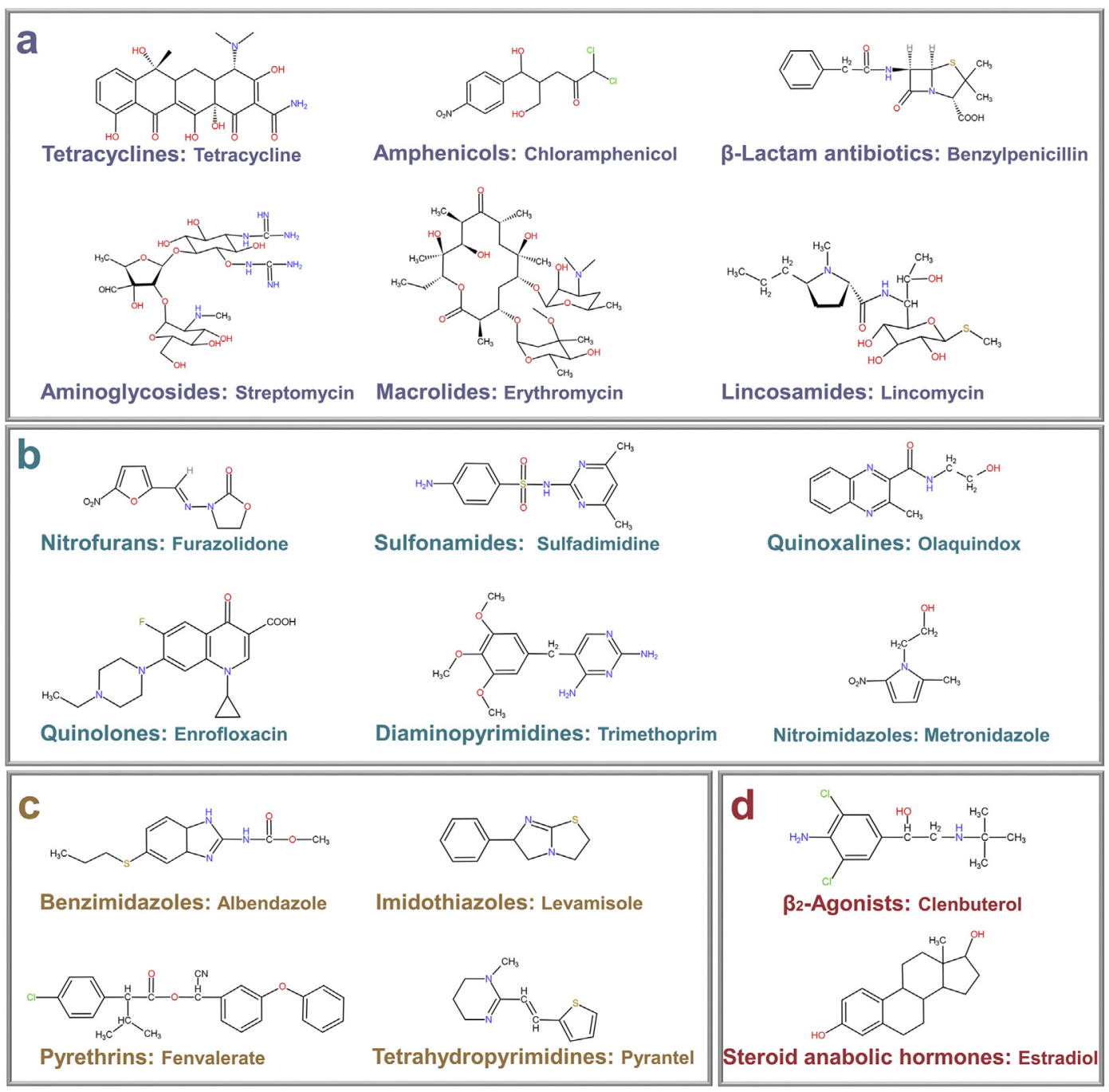

Fig. 1. Structures of some common veterinary drugs: (a) antibiotics, (b) synthetic antibacterials, (c) antiparasitic drugs, (d) growth-promoting agents.

methods, it is necessary to consider the physical and chemical properties of each sample type, and the state of the component to be tested. Some common pretreatment methods are summarized in Table 1 [34].

\section{Fluorescent nanoparticles}

Most of fluorescent nanoparticles used in the current detection of veterinary drug residues mainly include fluorescent microspheres, quantum dots, carbon dots, and metal nanoclusters. The design of fluorescent nanoparticles includes: (1) encapsulating fluorescent materials inside microspheres (Fig. 2a1, Fig. 2a2) [35,36]; (2) decorating fluorescent materials on the surface of microspheres (Fig. 2a3) [37]; (3) decorating an inorganic layer on the surface of quantum dots forming a core-shell structure or combing an organic functional layer (Fig. 2b) [38]; and (4) using fluorescent metal nanoclusters (Fig. 2c)

Table 1. Common food sample pretreatment for biosensors.

\begin{tabular}{lll}
\hline Methods & Advantages & Disadvantages \\
\hline Nitrogen blowing & (1) Concentration without oxygen & Suitable only for small volume or size of samples \\
& $\begin{array}{l}\text { (2) Time-saving } \\
\text { (3) Easy to operate and control }\end{array}$ & \\
Immunoaffinity chromatography (IAC) & High selectivity and stability & Complicated preparation of antibodies \\
Solid-phase extraction (SPE) & (1) Time-saving & (1) High cost of SPE columns \\
& (2) Achieving the trace separation & (2) Need professional operators \\
Rotary evaporation & (3) High selectivity and reproducibility & Boiling may occur, resulting in a loss of samples. \\
& (1) Flash distillation & \\
\hline
\end{tabular}




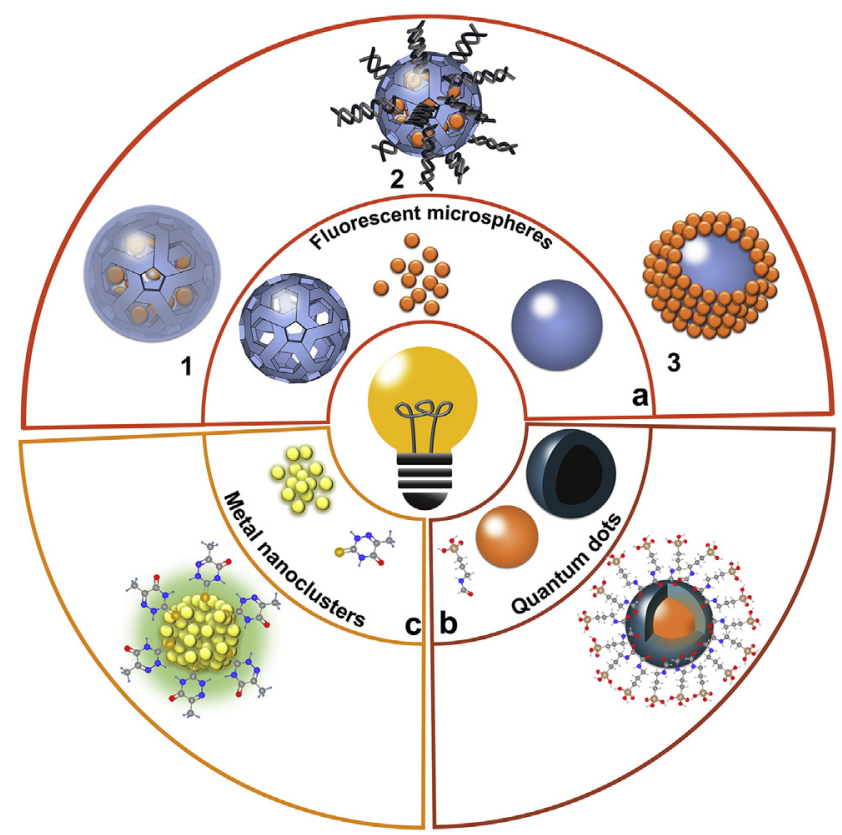

Fig. 2. Schematic illustration of various fluorescent nanoparticles for the detection of veterinary drugs: (a) fluorescent microspheres; (b) quantum dots; and (c) metal nanoclusters.

[39]. Some promising materials to prepare fluorescent microspheres are organic dyes such as fluorescein isothiocyanate and rhodamine $B$ [36] due to their high quantum yields and low cost. However, conventional organic dyes suffer from photobleaching and spectral overlapping [37]. In contrast, inorganic fluorescent materials, such as quantum dots, may overcome these challenges due to their excellent photostability, narrow and tunable emission spectra, and single excitation wavelength for different emitters [37].

\subsection{Fluorescent microspheres}

\subsubsection{Fluorescent microspheres labelled with organic dyes}

Fluorescence microspheres were manufactured by high-quality polystyrene and loaded with a variety of dyes (Fig. 2a1) [37]. The surface of fluorescent microspheres was easily functionalized (e.g. terminated by carboxylate or amine groups) to bind to targets. Due to strong $\pi-\pi$ interactions in the aggregated state, a high concentration of traditional organic dyes may sometimes suffer from aggregation-induced quenching [40]. Therefore, materials based on aggregation-induced emission (AIE) became a new generation of fluorescent labels because of the superior brightness in the aggregate state, larger Stokes shift, and higher relative quantum yield [41,42]. Compared to individual fluorophores, these fluorescent microspheres drastically amplify detection signals, which are particularly attractive for detection in food samples to overcome the background fluorescence.

\subsubsection{Fluorescent microspheres labelled with lanthanide complexes}

Lanthanide (e.g., $\mathrm{Tb}(\mathrm{III})$ and $\mathrm{Eu}(\mathrm{III})$ ) labelled nanomaterials have unique characteristics longlifetime emissions (up to $1 \mathrm{~ms}$ ) and large Stokes shifts (over $150 \mathrm{~nm}$ ) [43]. Therefore, interference from background fluorescence could be significantly reduced by means of a time delay that can be realized by time-gated luminescence (Fig. 3a) [44]. Lanthanide-doped up-conversion nanoparticles (UCNPs) demonstrated promising applications for sensitive detection of antibiotics [45]. With near-IR light of $980 \mathrm{~nm}$ for excitation, most background fluorophores cannot be excited while the UCNPs were efficiently excited, thus showing excellent optical features such as low phototoxicity and low autofluorescence background (Fig. 3b) [46].

\subsubsection{Fluorescent nanosilica}

Silica is a popular material for dye containment, and it has several advantages compared to polymerbased materials due to its high stability, low cost, and easy to synthesis. Fluorescent nanosilica are obtained by encapsulating fluorescent materials during sol-gel synthesis of silica nanoparticles. Silica can protect dyes such as ruthenium(II) complexes from oxidation and improve the photochemical stability and fluorescence intensity [47]. As a transition metal-based luminophore, $\mathrm{Ru}(\text { phen })_{3}^{2+}$ has potential applications in biosensors due to a high emission quantum yield, large Stokes shift, and long fluorescence lifetime [48]. Dyes can also be adsorbed into mesoporous silica nanoparticles (MSNs) and gated by the structure switching of aptamers after binding targets (Fig. 2a2) [49,50].

\subsection{Quantum dots}

Fluorescent semiconductor nanoparticles known as quantum dots (QDs) have received widespread attention with the promise to replace traditional organic dyes owing to their unique optical properties such as narrow emission bands and brightness [4]. They were used in immunoassays [51] and aptasensors [52] for veterinary drug detection. Most QDs have a core-shell structure (passivating QDs with an inorganic layer to improve its quantum yield and narrow the emission spectrum), such as CdSe QDs with a ZnS shell (ZnCdSe/ZnS) (Fig. 2b) [38]. 


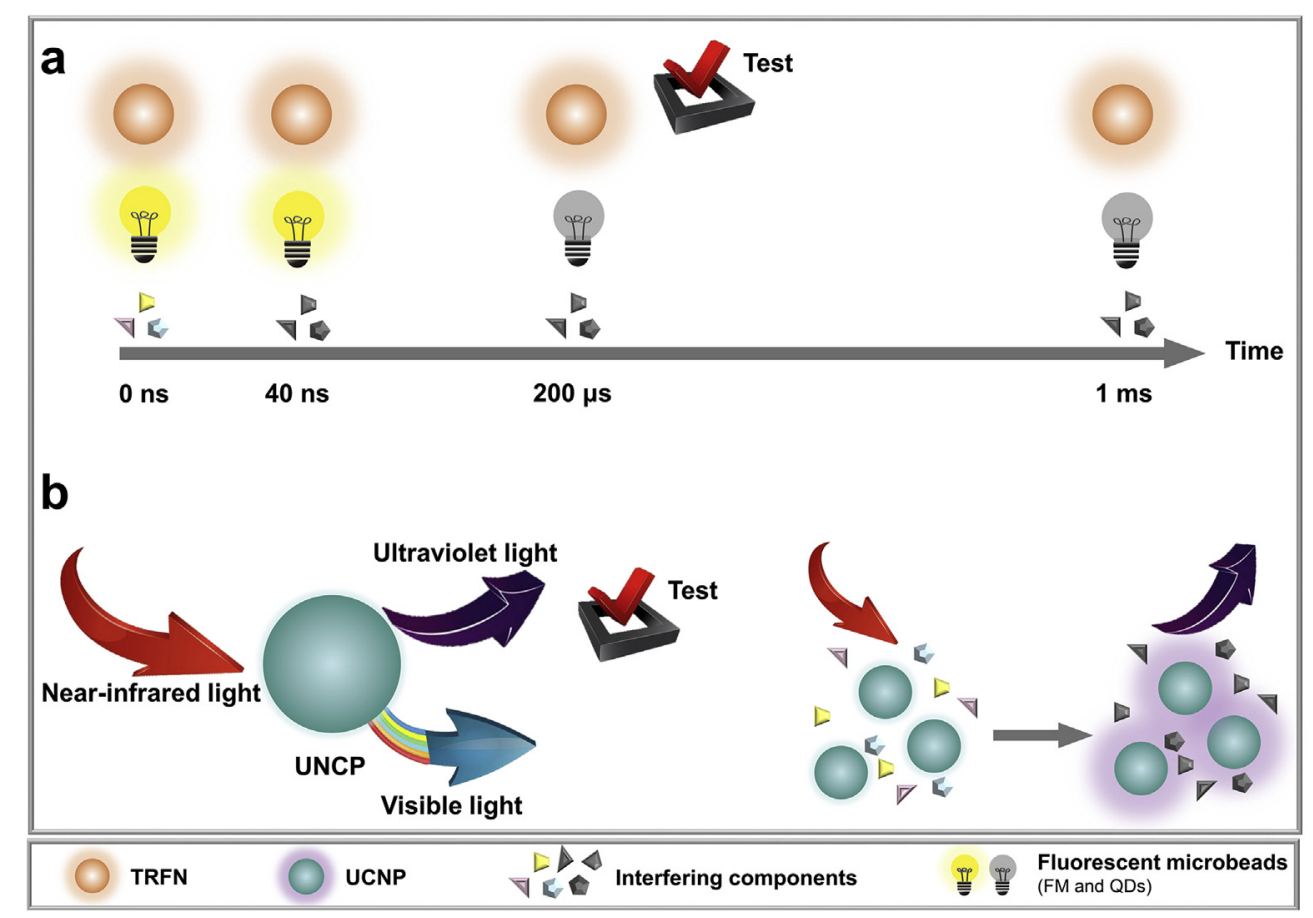

Fig. 3. (a) The extraction of useful fluorescence signals from nonspecific background fluorescence with the help of a time delay. (b) Ultraviolet to visible light emitted by UCNPs under near-infrared light excitation.

Most high-quality QDs were synthesized in organic solvents and then transferred to the aqueous phase. Moreover, the attachment of biological ligands such as antibodies and aptamers is not an easy task, which is generally achieved by coating QDs with a polymer or silica shell [53]. Another idea is to wrap hydrophobic QDs with an interdigitated lipid layer to transfer them to the aqueous phase [54], or liposome-encapsulated water-insoluble QDs [55].

\subsection{Carbon dots}

Carbon dots (CDs) are synthesized by mixing a carbon source (e.g. citrate) and a nitrogen source (e.g. ethylenediamine) in a hydrothermal condition [56]. Although the mechanism of CD fluorescence is still unclear, CDs have attracted much attention due to their high stability, bright fluorescence, low cytotoxicity, ease of synthesis, and convenient surface functionalization $[57,58]$.

\subsection{Noble metal nanoclusters}

Noble metal nanoclusters show potential applications in the design of sensors for veterinary drugs due to their excellent biocompatibility, good photostability, large Stokes shift, facile synthesis, low toxicity, and high catalytic activities [59]. For example, an immunoassay introduced 6-aza-2- thiothymine (ATT) and L-arginine (Arg) to synthesize Arg/ATT/AuNCs (ATT bound the guanidine group of Arg through hydrogen bonding) with strong green luminescence and good water solubility (Fig. 2c) [39]. Moreover, fluorescent silver nanoclusters (AgNCs) with size-dependent fluorescence emission were applied in bioassays, exhibiting excellent spectral and photophysical properties $[60,61]$.

\section{Immunosensing platforms}

Immunoassays are the main workhorse for detecting veterinary drug residues. Since veterinary drugs are mostly small molecules, competitive assays are the basic form of analysis. The concept of fluorescence immunoassay (FIA) was first proposed by the use of luminophore-labelled antibodies to locate antigens in tissues [62]. Combining fluorescence techniques with highly specific antibodies, FIA is high sensitive and accurate, realizing highthroughput automated detection [63]. In recent years, fluorescent immunosensing methods based on nanomaterials for veterinary drug detection are shown in Table 2.

\subsection{Fluorescence-linked immunoassays}

QDs, lanthanide chelates (such as europium), and UCNPs were used as fluorescent labels in FIA 


\begin{tabular}{|c|c|c|c|c|c|}
\hline Type & Analyte & Sample & Labelling Methods & Detection limit & Ref. \\
\hline \multirow[t]{3}{*}{ FLISA } & Quinolones & Milk & Carboxylate-modified QD655 labelled secondary antibody & $0.18 \mu \mathrm{g} \mathrm{L}^{-1}$ & [51] \\
\hline & Sulfonamides & & Avidin coated QD605-biotinylated primary antibody & $0.17 \mu \mathrm{g} \mathrm{L}^{-1}$ & \\
\hline & Melamine & & & $7.5 \mu \mathrm{g} \mathrm{L}^{-1}$ & \\
\hline FLISA & Sulfamethazine & Chicken muscle tissue & Carboxylate-modified QD655 labelled secondary antibody & $1.0 \mu \mathrm{g} \mathrm{L}^{-1}$ & [64] \\
\hline FLISA & Enrofloxacin & Chicken muscle tissue & Carboxylate-modified QD655 labelled secondary antibody & $2.5 \mu \mathrm{g} \mathrm{L}^{-1}$ & [65] \\
\hline \multirow[t]{2}{*}{ FLISA } & Tylosin & Edible animal tissues & Carboxylate-modified QD655 labelled secondary antibody & $0.02 \mu \mathrm{g} \mathrm{L}^{-1}$ & [66] \\
\hline & Tilmicosin & & & $0.04 \mu \mathrm{g} \mathrm{kg}^{-1}$ & \\
\hline FIA & Norfloxacin & Animal-derived foods & $\begin{array}{l}\text { Carboxyl-modified } \mathrm{NaYF}_{4}: \mathrm{Yb}_{4} \mathrm{Tm} \text { UNCPs labelled anti-target } \\
\mathrm{MAb}\end{array}$ & $0.01 \mu \mathrm{g} \mathrm{L}^{-1}$ & [67] \\
\hline FIA & Sulfaquinoxaline & Animal-derived foods & $\begin{array}{l}\text { Carboxyl-modified } \mathrm{NaYF}_{4}: \mathrm{Yb}, \mathrm{Tm} \text { UNCPs labelled anti-target } \\
\mathrm{MAb}\end{array}$ & $0.5 \mu \mathrm{g} \mathrm{kg}^{-1}$ & [68] \\
\hline FICA & Kanamycin & Milk & $\begin{array}{l}\mathrm{BHHCT}-\mathrm{Eu}^{3+} @ \mathrm{SiO}_{2} \text { labelled anti-target MAb (glutaraldehyde } \\
\text { crosslinking) }\end{array}$ & $0.85 \mu \mathrm{g} \mathrm{L}^{-1}$ & [35] \\
\hline FICA & Sulfamethazine & Honey & Carboxyl groups on AIEFM labelled anti-target MAb & $0.028 \mu \mathrm{g} \mathrm{L}^{-1}$ & [41] \\
\hline \multirow[t]{2}{*}{ FICA } & Enrofloxacin & Animal tissue & $\begin{array}{l}\text { Carboxylate-modified ZnCdSe/ZnS QDs labelled anti-target } \\
\mathrm{MAb}\end{array}$ & $5 \mu \mathrm{g} \mathrm{kg}^{-1}$ & [69] \\
\hline & & Milk & $\begin{array}{l}\text { Carboxylate-modified dyed polymer microsphere labelled anti- } \\
\text { target MAb }\end{array}$ & $10 \mu \mathrm{g} \mathrm{L}^{-1}$ & \\
\hline \multirow[t]{3}{*}{ FICA } & Ractopamine & Swine urine & $\begin{array}{l}\text { Carboxylate-modified time-resolved fluorescent nanobeads } \\
\text { labelled anti-target PAb }\end{array}$ & $7.2 \mathrm{ng} \mathrm{L}^{-1}$ & [70] \\
\hline & & & Fluorescent submicrospheres labelled anti-target PAb & $0.0147 \mu \mathrm{g} \mathrm{L}^{-1}$ & \\
\hline & & & Carboxylate-modified QDs labelled anti-target PAb & $0.0236 \mu \mathrm{g} \mathrm{L}^{-1}$ & \\
\hline FICA & Clenbuterol & Swine urine & $\begin{array}{l}\text { Carboxylate-modified Fluorescent magnetic nanobeads } \\
\text { labelled anti-target MAb }\end{array}$ & $0.22 \mu \mathrm{g} \mathrm{L}^{-1}$ & [71] \\
\hline FICA & Lincomycin & Milk, honey, beef & Carboxylate-modified FluoSpheres ${ }^{\circledR}$ labelled anti-target MAb & $0.69 \mu \mathrm{g} \mathrm{L}^{-1}$ & [72] \\
\hline FICA & Sulfamethazine & Milk & Carboxylate-modified FluoSpheres ${ }^{\circledR}$ labelled anti-target MAb & $0.11 \mu \mathrm{g} \mathrm{L}^{-1}$ & [73] \\
\hline FICA & Tilmicosin & Milk & Carboxylate-modified FluoSpheres ${ }^{\circledR}$ labelled anti-target MAb & $0.19 \mu \mathrm{g} \mathrm{L}^{-1}$ & [74] \\
\hline FICA & Enrofloxacin & Chicken meat & $\begin{array}{l}\mathrm{Ru}(\text { phen })_{3}{ }^{2+} \text {-doped FN labelled anti-target MAb (EDC } \\
\text { crosslinking) }\end{array}$ & $0.02 \mu \mathrm{g} \mathrm{L}^{-1}$ & [75] \\
\hline FICA & Oxytetracycline & Edible animal tissues & QDs labelled anti-target MAb (EDC crosslinking) & $0.44 \mu \mathrm{g} \mathrm{L}^{-1}$ & [76] \\
\hline FICA & Gentamicin & Milk & Carboxylate-modified QDs labelled anti-target PAb & $2 \mu \mathrm{g} \mathrm{kg}^{-1}$ & [77] \\
\hline FICA & Ractopamine & Swine urine and muscle tissue & $\begin{array}{l}\text { [FRET] Carboxylate-modified fluorescent polymer dots labelled } \\
\text { BSA AuNPs labelled anti-target MAb act as quencher }\end{array}$ & $0.16 \mu \mathrm{g} \mathrm{L}^{-1}$ & [78] \\
\hline FICA & Tetracycline antibiotics & Milk & [FRET] Carboxylate-modified ZnCdSe/ZnS QDs labelled OVA & $20 \mu \mathrm{g} \mathrm{L}^{-1}$ & [79] \\
\hline & & Animal muscle tissue & AuNPs labelled anti-target MAb act as quencher & $40 \mu \mathrm{g} \mathrm{kg}^{-1}$ & \\
\hline MLFIA & $\begin{array}{l}\text { Clenbuterol } \\
\text { Ractopamine }\end{array}$ & Swine urine & Carboxyl groups on Arg/ATT/AuNCs labelled anti-target MAb & $0.25 \mu \mathrm{g} \mathrm{L}^{-1}$ & [39] \\
\hline FTIACT & $\begin{array}{l}\text { Sulfonamide } \\
\text { Quinolone }\end{array}$ & Milk & $\begin{array}{l}\text { Liposome-encapsulated QDs labelled with hapten-carried } \\
\text { protein conjugates via N-succinimidyl 3-(2-pyridyldithio) } \\
\text { propionate }\end{array}$ & $\begin{array}{l}0.13 \mu \mathrm{g} \mathrm{L}^{-1} \\
0.062 \mu \mathrm{g} \mathrm{L}^{-1}\end{array}$ & [55] \\
\hline FICA & Macrolides & Raw milk & Carboxylate-Modified FluoSpheres $®$ labelled antibody & $0.13 \mu \mathrm{g} \mathrm{L}^{-1}$ & [80] \\
\hline
\end{tabular}




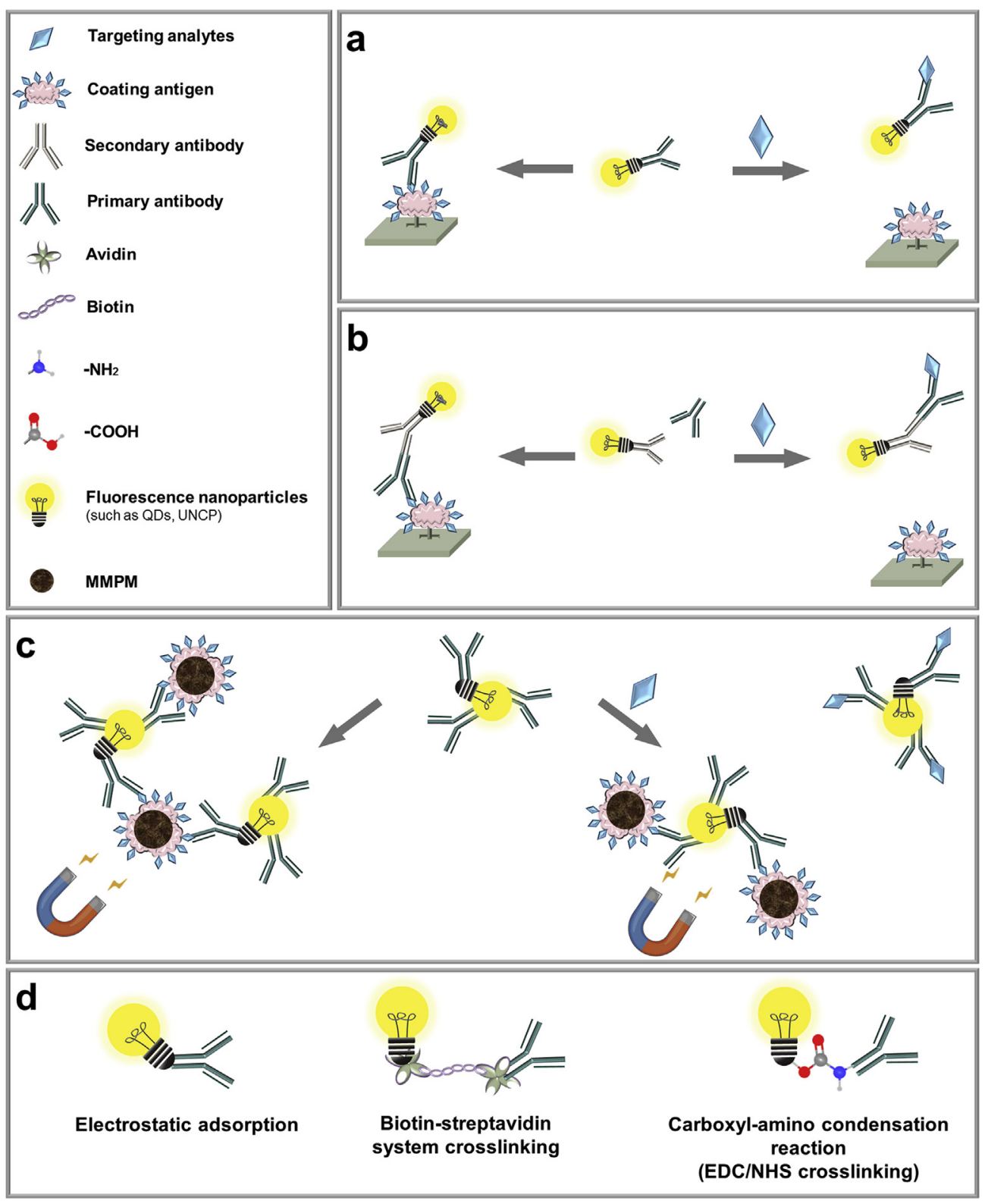

Fig. 4. Schematic illustration of fluorescence-linked immunoassays for the detection of veterinary drugs: (a) direct competition; (b) indirect competition; and (c) combined with immunomagnetic separation. (d) Bioconjugation methods between antibodies and fluorescent nanoparticles.

(Fig. 4). Fluorescent nanoparticles labelling of antibodies was mainly achieved by means of biotinstreptavidin binding, electrostatic adsorption, and the amino-carboxyl condensation reaction (Fig. 4d) $[51,64,68]$. The amino-carboxyl condensation reaction is typically facilitated by EDC/NHS coupling (EDC: 1-ethyl-3-[3(dimethylamino)propyl]carbodiimide; NHS: N-hydroxysuccinimide) [68].

\subsubsection{Direct competition}

As depicted in Fig. 4a, a fluorescence-linked immunosorbent assay (FLISA) was developed using primary antibodies modified with fluorescent nanoparticles. In the absence of targets, the labelled primary antibodies bound to the coating antigens on a microplate. In the presence of targets, the targets and the coating antigens competed to bind with the labelled primary antibodies, and the labelled primary antibodies remained in solution were washed away, leading to a lower fluorescence on the microplate (Fig. 4a).

\subsubsection{Indirect competition}

As described in Fig. $4 \mathbf{b}$, indirect competition FLISA used secondary antibodies labelled with fluorescent nanoparticles to bind to primary 
antibodies. Compared to the direct competitive assay, the labelled secondary antibody was added after adding primary antibodies. QDs were usually linked to secondary antibodies via the biotin-streptavidin conjugation [51] or the amino-carboxyl condensation reaction [64-66]. In particular, the unique binding characteristics of biotin/avidin (avidin as a bridge to the secondary antibody) decreased the adverse effect on the cross-reactivity of primary antibodies with directly conjugated QD. A FLISA was developed for the simultaneous determination of sulfonamides, quinolones, and melamine in milk [51].

\subsubsection{Immunomagnetic separation}

FIA can also be combined with magnetic separation to further improve sensitivity (Fig. 4c). In the absence of targets, primary antibodies labelled with fluorescent nanoparticles could bind coating antigens (immobilized on magnetic polystyrene microspheres), and a strong fluorescence is detected in the system. In the presence of targets, targets bound to the labelled primary antibodies and are removed via magnetic separation, thus decreasing the fluorescence. The strategy was employed to detect sulfaquinoxaline in animal-derived foods, with a LOD of $0.5 \mu \mathrm{g} \mathrm{kg}^{-1}$ [68].

\subsection{Fluorescent immunochromatographic assay (FICA)}

\subsubsection{Traditional fluorescent lateral flow immunoassay}

Typical immunoassays require multiple washing and incubation steps. Although highly sensitive, it is difficult to apply these methods outside a lab. Immunochromatographic assay was developed to solve this problem by integrating the assays on a simple flow device, and the best-known example is the pregnancy test strips [81]. Fluorescent immunochromatographic assay (FICA) was also developed to detect veterinary drugs in food samples with a competitive immunoassay principle.

As shown in Fig. 5a, coating antigens were pinned to the test line of the strip to bind primary antibodies labelled with fluorescent nanomaterials, and secondary antibodies were pinned to the control line to capture the unbound primary antibodies (a positive control line indicates that the test result is valid). Without a target, the labelled primary antibodies can bind with the antigens coated on the test line, and the fluorescence is strongest. With the increase of target concentration, more targets bind to the labelled primary antibodies to decrease the signal.
A study compared FICAs based on anti-target polyclonal antibody labelled with QDs, fluorescent submicrospheres and time-resolved fluorescent nanobeads (TRFNs) for ractopamine detection in

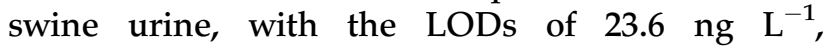
$14.7 \mathrm{ng} \mathrm{L}^{-1}, 7.2 \mathrm{ng} \mathrm{L}^{-1}$, respectively [70]. With a $200 \mu$ s delay time in fluorescence measurement, TRFN-FICA had the lowest LOD, shortest detection time and widest linear range. Therefore, the above three FICAs are also suitable for the detection of veterinary drug residues in food samples, and the use of TRFNs recommended. FICA is well suited for the competitive format and has a broader prospect for veterinary drug detection in practical application with the obvious advantages (time-saving, easy operation, high sensitivity and specificity).

\subsubsection{Combined with immunomagnetic separation}

FICA can also combine with immunomagnetic separation (Fig. 5b). Instead of just using a fluorophore label, primary antibodies are labelled on a fluorescent magnetic nanobead (FMNB), which is also used for sample purification. The principle of the subsequent detection process is similar to Fig. 5a. A FICA combined with immunomagnetic separation was established to detect the clenbuterol residue in swine urine with the LOD of $0.22 \mathrm{ng} \mathrm{mL}^{-1}$ [71], four times lower than that of traditional colloidal gold-based ICA. The magnetic separation can minimize the effect of the sample matrix in veterinary drug detection to boost sensitivity and specificity.

\subsubsection{Multi-component analysis}

FICAs can also be used to simultaneously analyze multiple components or more than one group of veterinary drugs, and these are called multiplex lateral flow immunoassays (MLFIA). As shown in Fig. 5c, two test lines (test line 1 and 2) are marked with coating antigens of two different targets. In the absence of targets, the coating antigens on the test line bind with the antibodies labelled with fluorescent nanoparticles and show the highest fluorescence intensity. In the presence of one or both targets, targets bind to antibodies labelled with fluorescent nanoparticles, and the fluorescence of the corresponding test line (test line 1 and/or test line 2) decrease because of the competitive binding. The fluorescence intensity ratio of the test lines to the control line is used to quantitatively measure binding and offset the inherent heterogeneity of MLFIA. A MLFIA was developed for the simultaneous detection of clenbuterol and ractopamine in swine urine [39]. 


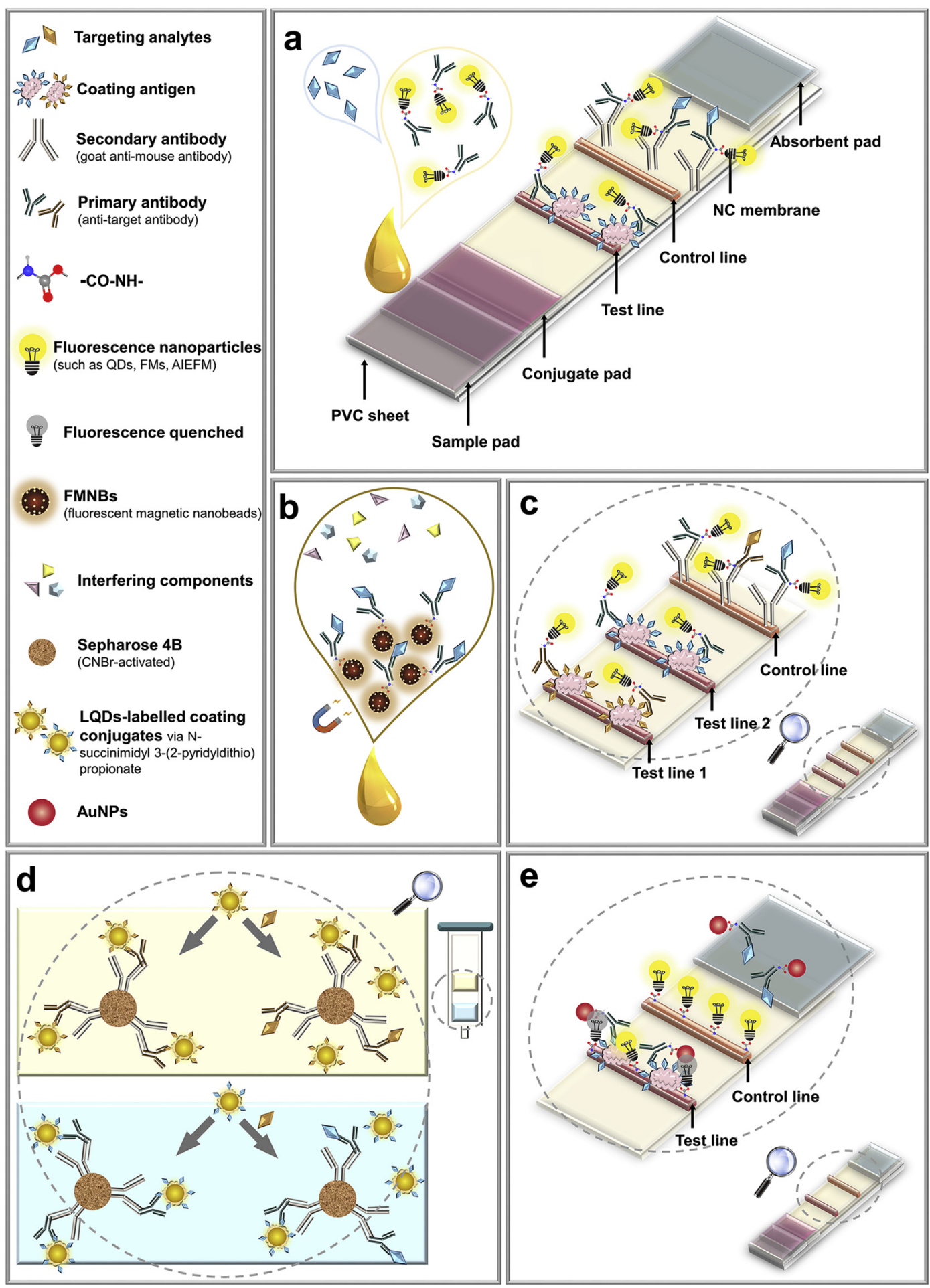

Fig. 5. Schematic illustration of fluorescent lateral flow immunoassay for veterinary drug detection: (a) traditional assay (figure created based on ref. [41]); (b) combined with immunomagnetic separation (figure created based on ref. [71]); (c) multiplex lateral flow immunoassay (figure created based on ref. [39]); (d) multiplex flow-through immunoaffinity chromatography test (figure created based on ref. [55]); (e) base on FRET (figure created based on ref. [79]).

A multiplex flow-through immunoaffinity chromatography test (FTIACT) is shown in Fig. 5d. In this design, the secondary antibody is labelled on sepharose $4 \mathrm{~B}$ beads, and the primary antibodies are attached to the secondary antibodies. The targets compete with liposome-encapsulated QDs-labelled targets to bind with the primary antibodies. A multiplex FTIACT was developed for the detection 


\begin{tabular}{|c|c|c|c|c|c|}
\hline Strategy & Analyte & Sample & Method & Detection limit & Ref. \\
\hline \multirow{11}{*}{$\begin{array}{l}\text { Nanomaterials as } \\
\text { fluorescence } \\
\text { quenchers }\end{array}$} & Oxytetracycline & Honey, water & FRET from thiol-Aptamer modified AgNCs to target. & $0.046 \mu \mathrm{g} \mathrm{L}^{-1}$ & [61] \\
\hline & Sulfadimethoxine & Milk & FRET from Aptamer-FAM to CPNBs. & $10 \mu \mathrm{g} \mathrm{L}^{-1}$ & [84] \\
\hline & Oxytetracycline & Lake water & FRET from Aptamer-FAM to rGO. & $4.6 \mu \mathrm{g} \mathrm{L}^{-1}$ & [85] \\
\hline & Tetracycline & Milk & $\begin{array}{l}\text { FRET from Aptamer-VS } 2 \text { QDs to } \mathrm{MoS}_{2} \text { nanosheets. } \\
\text { (amino-carboxyl condensation reaction between } \mathrm{VS}_{2} \\
\text { QDs and aptamer) }\end{array}$ & $0.06 \mu \mathrm{g} \mathrm{L}^{-1}$ & [86] \\
\hline & Tetracycline & Animal-derived food & $\begin{array}{l}\text { FRET from Aptamer-N-GQDs to CoOOH. (amino- } \\
\text { carboxyl condensation reaction between N-GQDs } \\
\text { and aptamer) }\end{array}$ & $0.95 \mu \mathrm{g} \mathrm{L}^{-1}$ & [87] \\
\hline & Oxytetracycline & Tap water, river & FRET from Aptamer-FAM to GO hydrogel. & $25 \mu \mathrm{g} \mathrm{L}^{-1}$ & [88] \\
\hline & Kanamycin & Milk, animal-derived food & $\begin{array}{l}\text { FRET from Aptamer-CDs to } \mathrm{MoS}_{2} \text { nanosheets. } \\
\text { (amino-carboxyl condensation reaction between CDs } \\
\text { and aptamer) }\end{array}$ & $532.95 \mu \mathrm{g} \mathrm{L}^{-1}$ & [89] \\
\hline & Kanamycin & Milk & FRET from Aptamer-FAM to rGO. & $0.4845 \mathrm{ng} \mathrm{L}^{-1}$ & [90] \\
\hline & Florfenicol & Milk & FRET from Aptamer-ATTO647N to GO. & $2.06 \mu \mathrm{g} \mathrm{L}^{-1}$ & [91] \\
\hline & Oxytetracycline & Spiked milk and tablets & $\begin{array}{l}\text { FRET from oxytetracycline-Eu }{ }^{3+} \text { to AgNPs. } \\
\text { (electrostatic adsorption between targets and } \mathrm{Eu}^{3+} \text { ) }\end{array}$ & $0.874 \mu \mathrm{g} \mathrm{L}^{-1}$ & [92] \\
\hline & Kanamycin A & Milk & FRET from Aptamer-FAM to AuNPs & $0.145 \mu \mathrm{g} \mathrm{L}^{-1}$ & [93] \\
\hline \multirow[t]{3}{*}{ Label-free sensors } & Enrofloxacin & Milk & FRET from targets to GO. & $1.33 \mu \mathrm{g} \mathrm{L}^{-1}$ & [94] \\
\hline & Ofloxacin & Milk & FRET from Rhodamine B to AuNPs. & $1.66 \mu \mathrm{g} \mathrm{L}^{-1}$ & [95] \\
\hline & Kanamycin & Milk & IFE from CDs to AuNPs. & $8.72 \mu \mathrm{g} \mathrm{L}^{-1}$ & [96] \\
\hline $\begin{array}{l}\text { Direct detection } \\
\text { [Splitting aptamers] }\end{array}$ & Kanamycin & Human serum & $\begin{array}{l}\text { One DNA split was labelled with CuS nanoparticle } \\
\text { by amino-carboxyl condensation reaction, and the } \\
\text { other was coupled with paramagnetic particles } \\
\text { (PMPs) by biotin-streptavidin system crosslinking. }\end{array}$ & $12.597 \mathrm{ng} \mathrm{L}^{-1}$ & [97] \\
\hline \multirow[t]{4}{*}{$\begin{array}{l}\text { Indirect competitive } \\
\text { [Strand-displacement } \\
\text { strategy] }\end{array}$} & Kanamycin & Milk & $\begin{array}{l}\text { FRET from QDs-SSB to Aptamer-BHQ } \text { (amino- }_{1} \text { (arboxyl condensation reaction between QDs and } \\
\text { cSB) }\end{array}$ & $0.006 \mu \mathrm{g} \mathrm{L}^{-1}$ & [52] \\
\hline & Oxytetracycline & Milk & FRET from FAM (labelled on CS) to GO. & $4.6043 \mathrm{mg} \mathrm{L}^{-1}$ & [98] \\
\hline & Oxytetracycline & Milk & $\begin{array}{l}\text { LRET from } \mathrm{NaYF}_{4}: \mathrm{Yb}, \mathrm{Tm} \text { UCNPs to SYBR Green I. } \\
\text { (amino-carboxyl condensation reaction between } \\
\text { UCNPs and aptamer) }\end{array}$ & $0.054 \mu \mathrm{g} \mathrm{L}^{-1}$ & [99] \\
\hline & Kanamycin & Milk, serum & FRET from FAM (labelled on CS) to AuNPs. & $0.156 \mu \mathrm{g} \mathrm{L}^{-1}$ & [100] \\
\hline
\end{tabular}




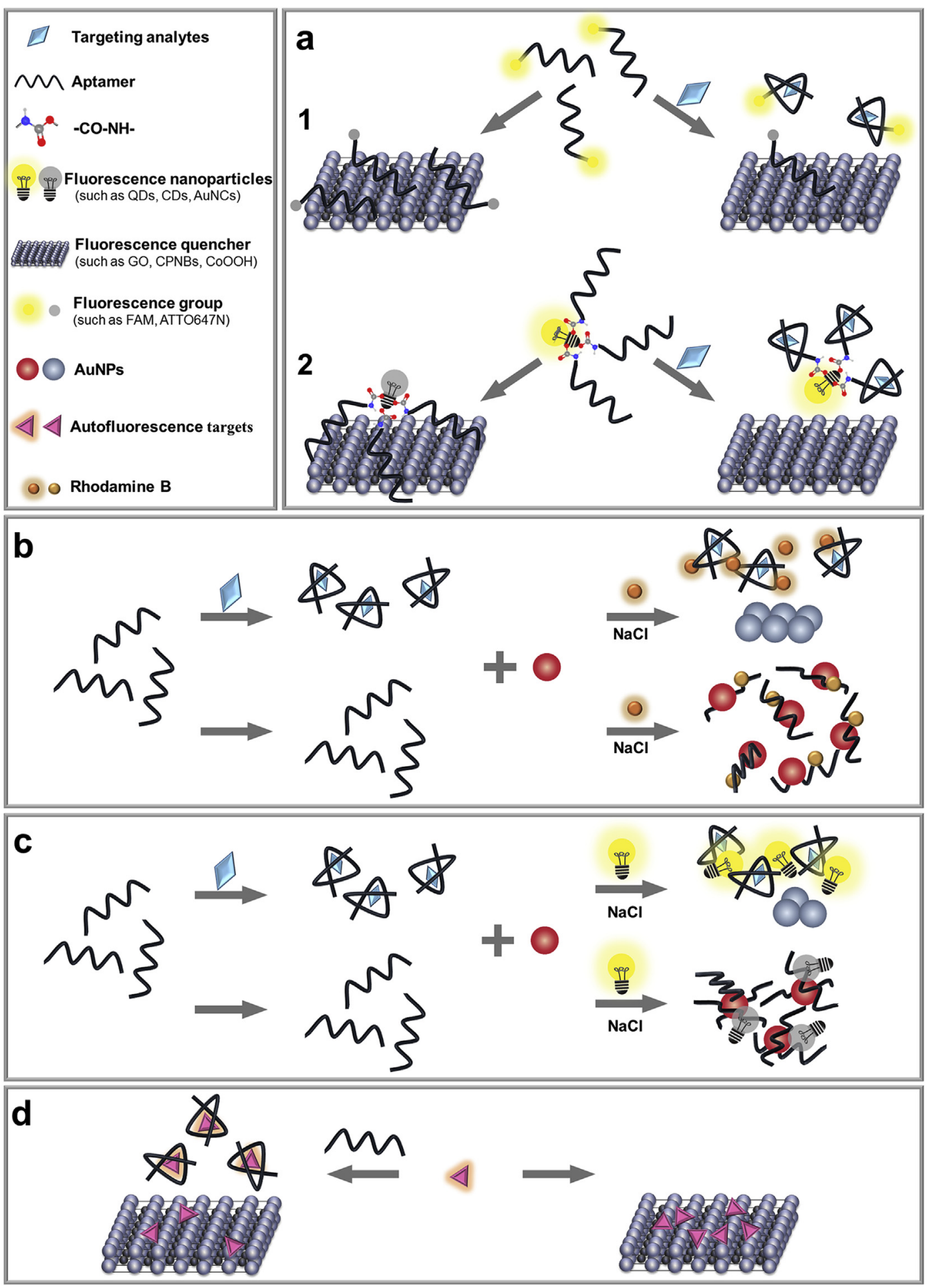

Fig. 6. Schematic illustration of assays based on nanomaterials as fluorescence quenchers and label-free sensors for the detection of veterinary drugs. (a) Labelled type (figure created based on ref. [84,86]). (b) Label-free assay base on FRET from rhodamine B to AuNPs (figure created based on ref. [95]). (c) Label-free assay base on IFE from CDs to AuNPs (figure created based on ref. [96]). (d) Label-free assay base on the autofluorescence of target (figure created based on ref. [94]). 
of 14 sulfonamides and 13 quinolones residues in milk, with LODs of $1 \mathrm{ng} \mathrm{mL}^{-1}$ and $0.5 \mathrm{ng} \mathrm{mL}^{-1}$ for the sulfonamides and quinolones, respectively [55]. Multiplex FTIACT is promising for qualitative and quantitative detection of multiple veterinary drugs in food samples.

\subsubsection{Assays based on fluorescence resonance energy transfer}

As shown in Fig. 5e, fluorescence resonance energy transfer (FRET) has also been used in FICA. In this example, the fluorescent polymer dots-labelled BSA was the fluorophore, and AuNP-labelled primary antibody (anti-target MAb) was the quencher. In the absence of targets, AuNP-labelled primary antibodies bound with the coating antigen on the test line, and the fluorescence of QDs (fixed on the test line) was quenched by AuNPs at this time. After adding targets, the FRET effect was reduced, and the fluorescence of the test line was high. This way, more fluorescence signal was produced in this competitive assay along with the increase in target concentration. This method allowed quantities analysis of ractopamine and tetracycline antibiotics in animal muscle tissues, with the LODs of $0.16 \mathrm{ng} \mathrm{mL}^{-1}$ [78] and $40 \mu \mathrm{g} \mathrm{kg}^{-1}$ [79], respectively.

\section{Aptamer-based assays}

Traditional immunoassays based on antigen-antibody recognition have a high production cost and antibodies may suffer from large batch-to-batch variation. Meanwhile, labelling of fluorophores is also complex and difficult to be achieve site-specific labeling. Nucleic acid aptamers are similar to antibodies in selective targets binding, and they are particularly suitable for the binding of small molecular targets. Aptamers are isolated from an enriched nucleotide library by the SELEX (systematic evolution of ligands by exponential enrichment) method [82]. Aptamers have been widely used in environmental monitoring, food safety, and veterinary drug detection due to their simple and rapid synthesis, easy modification, low cost, high affinity, and high stability [83]. In recent years, aptamer-based fluorescent biosensors using nanomaterials for veterinary drug detection are shown in Table 3.

\subsection{Nanomaterials as fluorescence quenchers}

As shown in Fig. 6a, adsorption of a fluorescently labelled aptamer on some nanomaterials can result in fluorescent quenching. In the presence of target analytes, the aptamer is desorbed to enhance the fluorescence signal. Common fluorescent quenchers including coordination polymer nanobelts (Fig. 6a1) [84], $\mathrm{VS}_{2}$ QDs, molybdenum (IV) disulfide $\left(\mathrm{MoS}_{2}\right)$ nanosheets (Fig. 6a2) [86], and other examples shown in Table 3. Sometimes, even the target molecules can act as fluorescent quenchers, such as oxytetracycline [61] and oxytetracycline- $\mathrm{Eu}^{3+}$ [92].

It is worth noting that not all nanomaterials are equal. For example, it is very difficult to desorb aptamers from AuNPs due to an extremely strong adsorption affinity. Recent work showed that only less than $5 \%$ of adsorbed aptamers could be desorbed by target molecules, and even this small number of desorbed aptamers were likely due to nonspecific displacement instead of specific aptamer binding [82,101,102]. An aptasensor for the detection of kanamycin was developed by quenching of the FAM-labelled aptamer on AuNPs [93]. In

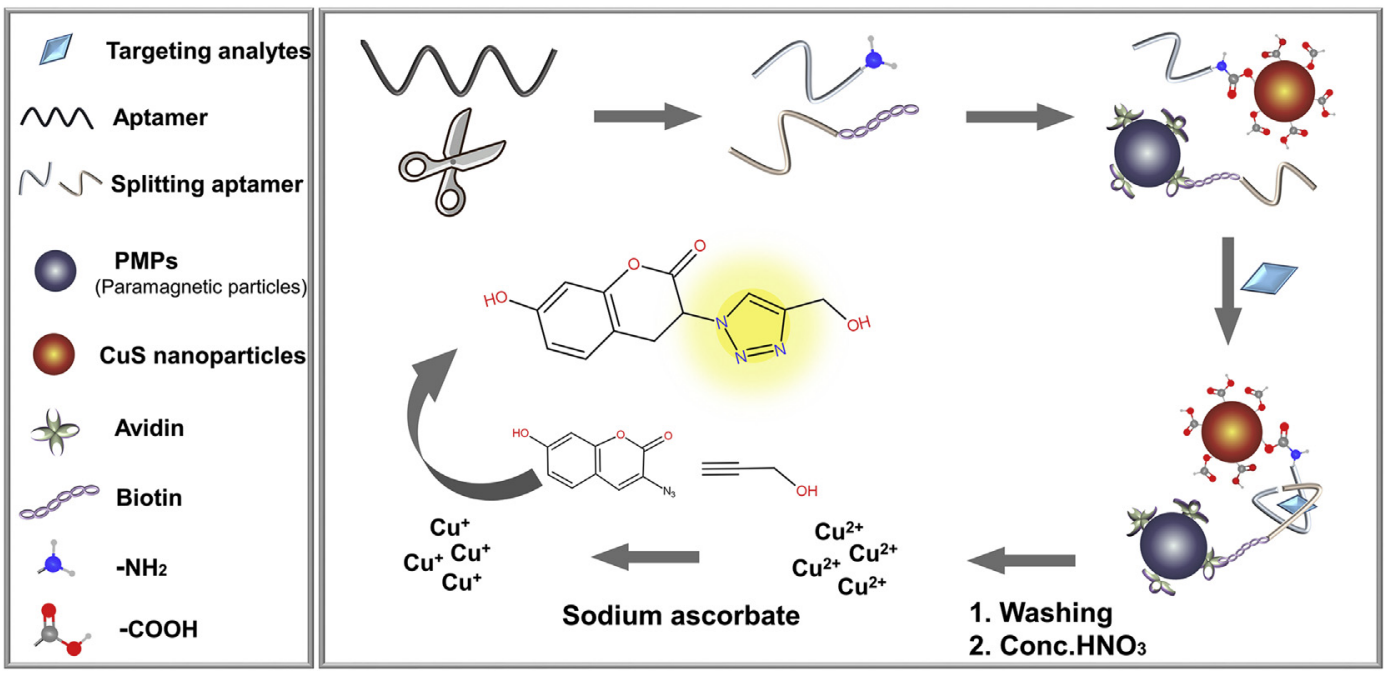

Fig. 7. Schematic illustration of assays based on the splitting aptamers for the detection of veterinary drugs (figure created based on ref. [97]). 


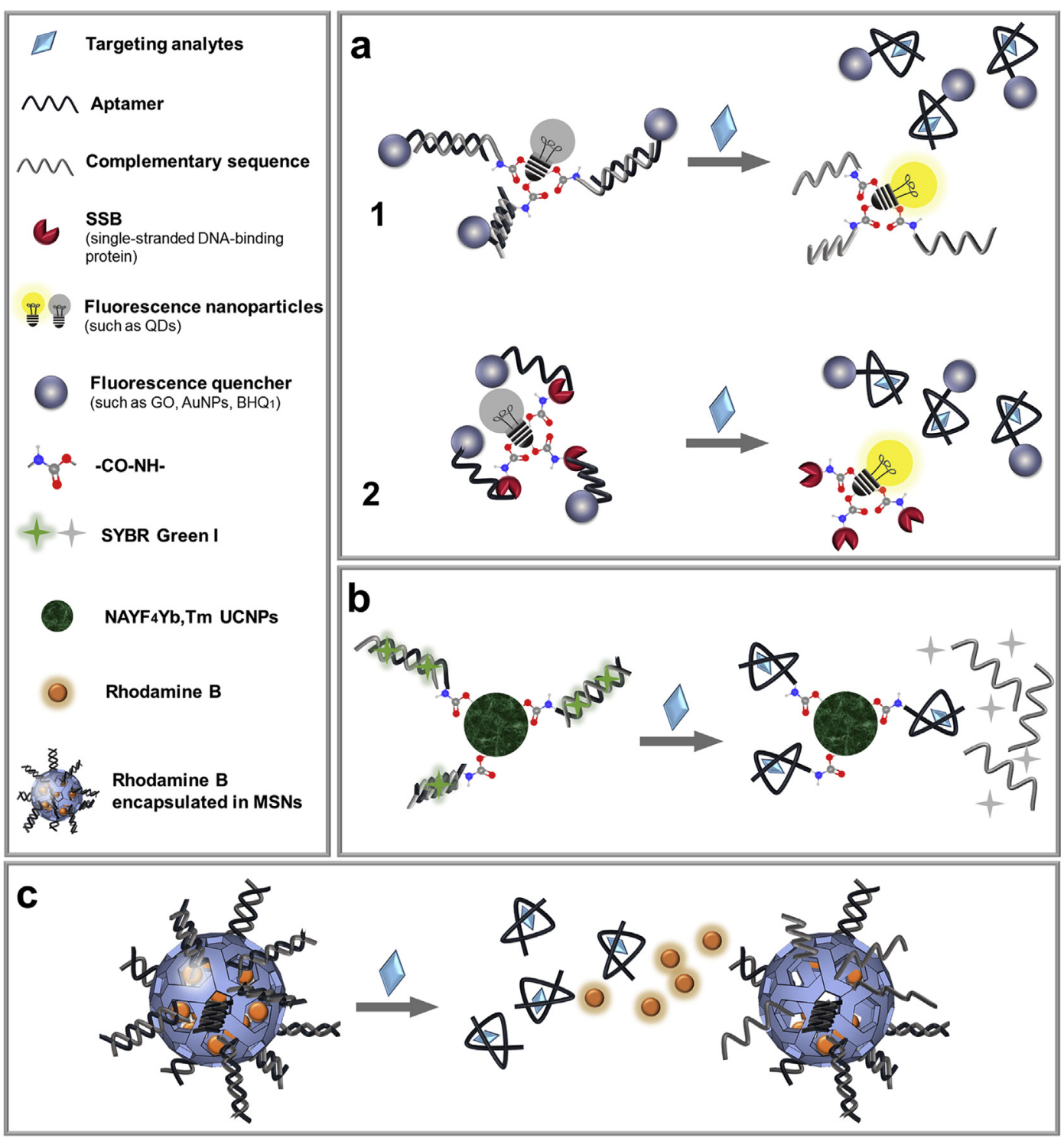

Fig. 8. Schematic illustration of assays based on the structure-switching aptamer strategies for the detection of veterinary drugs. (a) Assays based on FRET (figure created based on ref. [52,98]). (b) Assays based on LRET (figure created based on ref. [99]). (c) Assays based on gated release of dyes from MSNs (figure created based on ref. [36]).

this case, the saturated fluorescence enhancement was only about $25 \%$. Although the authors did not show the total adsorbed DNA, based on our results, 10 -fold or more enhancement can be readily achieved if all the adsorbed DNAs were desorbed (e.g. by adding $\mathrm{KCN}$ ) [101,103].

\subsection{Label-free sensors}

Since label-free sensors do not require a covalent label, they are more cost-effective. As shown in Fig. $6 \mathrm{~b}$, a label-free strategy is described. In the absence of targets, AuNPs remain dispersed due to absorbed aptamers in a high concentration of $\mathrm{NaCl}$, and the dispersed AuNPs could reduce the fluorescence intensity of Rhodamine $B$ by the inner-filter effect. In the presence of targets, the aptamers were expected to bind to targets and thus could not be absorbed on AuNPs. Therefore, the AuNPs were exposed and aggregated in the $\mathrm{NaCl}$ solution, resulting in a high fluorescence intensity [95]. Aptasensor for ofloxacin detection in milk was established with a LOD of $1.66 \mu \mathrm{g} \mathrm{L}^{-1}$ [95].

Similarly, a strategy developed using the inner filter effect using fluorescence of CDs (Fig. 6c). An aptasensor was developed for kanamycin detection in milk with a LOD as low as $18 \mathrm{nM}$ [96]. It needs to be noted that some targets (such as kanamycin) are 
also likely to compete with its aptamer for the adsorption sites on the AuNPs, meaning that preadsorbed kanamycin might inhibit the adsorption of its aptamer [103,104]. Thus, it is necessary to conduct control experiments with random DNA that cannot bind target molecules to ensure the proposed sensing mechanism.

Moreover, an interesting aptasensor was developed by the autofluorescence of the target, enrofloxacin (Fig. 6d). In the absence of the aptamer, the fluorescence of enrofloxacin was quenched by adsorption on graphene oxide (GO). In the presence of the aptamer, the enrofloxacin-aptamer complex had a lower affinity for GO and the fluorescence intensity increased. This aptasensor was developed to detect enrofloxacin in milk with a calculated LOD of $3.7 \mathrm{nM}$ [94].

\subsection{Sensors based on split aptamers}

An interesting feature of aptamers is its programmability. For example, an aptamer can be split into two pieces and binding can still occur when both pieces are present. However, this type of splitting is more difficult for antibodies. Split aptamers provide additional methods for sensing. As shown in Fig. 7, binding of targets can shorten the distance of the two split aptamer fragments, resulting in a fluorescence signal change. Using the detection of kanamycin as an example, its split aptamer fragments were respectively labelled with CuS nanoparticles and biotin which could couple with streptavidin coated paramagnetic particles. The presence of kanamycin allowed magnetic separation of the $\mathrm{CuS}$ nanoparticles, which were then dissolved by concentrated nitric acid. The generated $\mathrm{Cu}(\mathrm{II})$ ions were reduced to $\mathrm{Cu}(\mathrm{I})$ by sodium ascorbate to act as a catalyst for the click chemistry and produce a fluorophore. The detection range was 0.04-20 nM [97].

\subsection{Sensors based on structure-switching strategies}

Since aptamers are also a piece of DNA, they can bind not only their target molecules but also their complementary DNA. Structure-switching aptamer based sensors are developed based on the competition of these two binding events [105]. The structure-switching strategy can be combined with FRET to avoid the centrifugation step. Both GO [98] or AuNPs [100] ere used as quenchers (Fig. 8a1). The fluorescent assays for oxytetracycline and kanamycin detection in milk were presented with LODs of $0.01 \mathrm{mM}$ [98] and $321 \mathrm{pM}$ [100], respectively. Furthermore, researchers employed the single- 


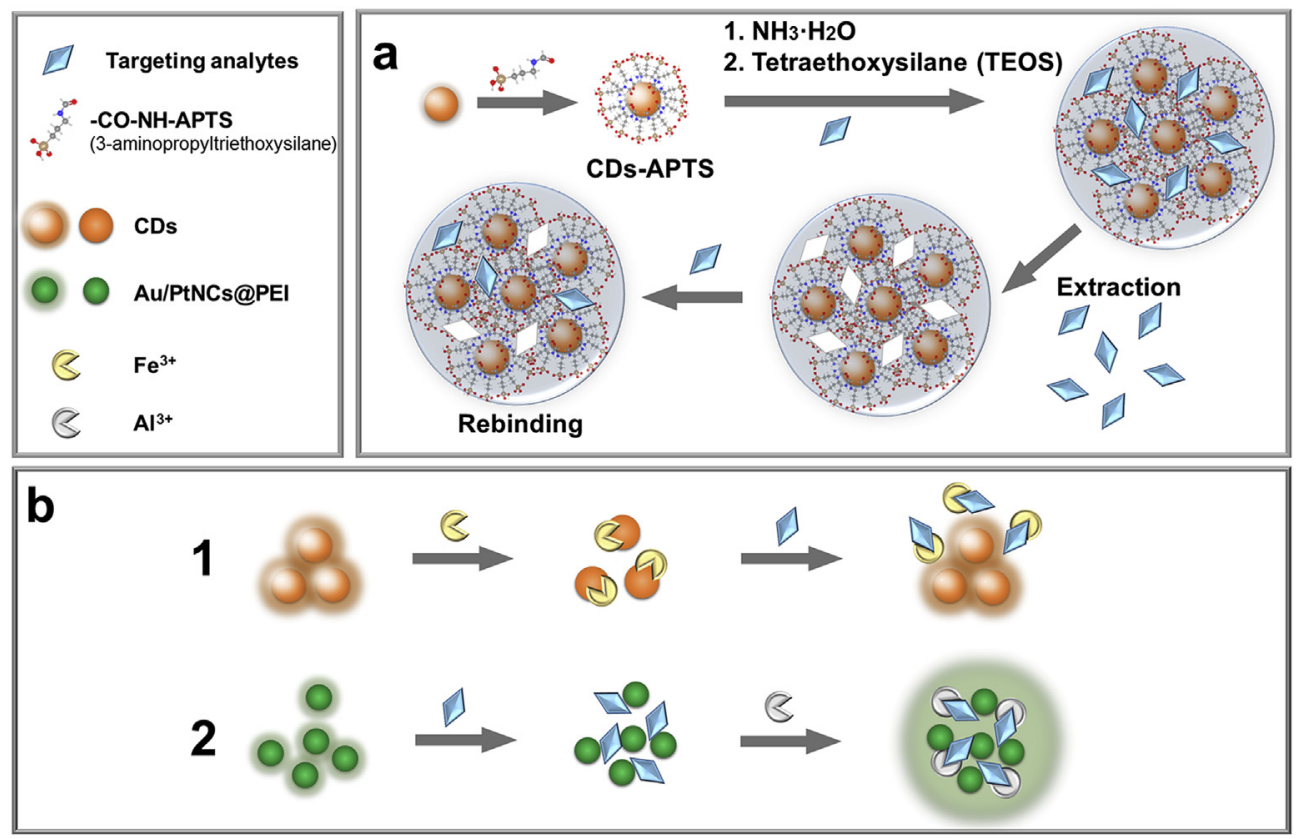

Fig. 9. (a) Schematic illustration of target recognition based on MIPs for the detection of veterinary drugs (figure created based on ref. [109]). (b) Schematic illustration of target recognition based on metal coordination for the detection of veterinary drugs (figure created based on ref. [112,113]).

stranded DNA-binding protein (SSB) as an alternative to the complementary chain. SSB was covalently linked to the fluorescent graphene oxide quantum dots (GOQDs) by amino-carboxyl condensation reaction (Fig. 8a2). A BHQ quencher)-labeled aptamer was added to bind to the GOQDs via the SSB resulting in quenched fluorescence. Upon addition of the target, kanamycin, the quencher-labeled aptamer would dissociate from the GOQDs resulting in increased fluorescence. This method showed a LOD of $6 \mathrm{pg} \mathrm{mL}^{-1}$ kanamycin in milk [52].

On top of that, a biosensor using the luminescence resonance energy transfer (LRET) from UCNPs to SYBR Green I dye was established [99]. As shown in Fig. $8 b$, the aptamers were hybridized with their complementary strands and covalently linked to UCNPs. SYBR Green I was inserted into the formed dsDNA duplex, and the fluorescence of the UCNPs could transfer to it. In the presence of the target oxytetracycline, SYBR Green I was released and the UCNP luminescence increased. Under optimal conditions, the LOD for oxytetracycline detection in

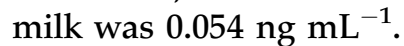

Another type of the label-free structure-switching strategy is shown in Fig. 8c, where an aptamer was hybridized to amine-modified MSNs to block the pores, which were filled with rhodamine B [36]. In the presence of the target kanamycin, a significant increase in fluorescence intensity was observed due to the separation of aptamers from complementary strands and the leakage of Rhodamine B. The saturated change of fluorescence signal was however only $12 \%$. Both kanamycin and streptomycin showed a similar fluorescence increase, although this aptamer should only bind the former [106]. Therefore, nonspecific interactions could not be ruled out.

\section{Target recognition based on molecularly imprinted polymers}

In addition to aptamers and antibodies, molecular imprinted polymers (MIPs) represented another general method for molecular recognition [107]. MIP referred to the polymerization of selected functional monomers around a target molecule to imprint the shape of the target. After washing away the target, the resulting polymer cavity can selectively rebind the target [108]. Production of highly stable MIP is cost-efficient and can be made in bulk quantity, although the selectivity is in general not as good as that of antibodies and aptamers. Some fluorescent biosensors using MIPs and other nanomaterials to detect veterinary drugs in food samples are shown in Table 4.

As shown in Fig.9a, a CDs@MIP composite was synthesized by grafting MIP onto the surface of 3- 
aminopropyltriethoxysilane (APTS)-modified CDs by amino-carboxyl condensation reaction. The CD@MIPs showed a high extraction efficiency for the targets tetracycline [109]. The concentration of tetracycline was quantitatively analyzed by fluorescence quenching (the fluorescent intensity of CDs@MIPs was quenched with increasing concentration of tetracycline) with a LOD of $5.48 \mathrm{nM}$ in milk [109].

Another biosensor was developed for enrofloxacin and danofloxacin detection with the LODs of 0.28 and $0.08 \mu \mathrm{M}$ in milk, respectively [110]. As fluoroquinolones, these two drugs exhibited inherent fluorescence, which was widely used for their quantitation [111]. The free drug molecules had a low polarization value, while the polarization increased upon binding to their MIPs, allowing their detection.

\section{Target recognition based on metal coordination}

Many drug molecules have metal binding groups and such metal coordination interactions have also been harnessed to develop fluorescent sensors when combined with nanomaterials. In some systems, both metal ions and target molecules can affect the fluorescence of nanomaterials. As shown in Fig. 9b1, the catechol groups on the surface of water-soluble fluorescent $\mathrm{CDs}$ can bind $\mathrm{Fe}^{3+}$ ions and render fluorescence quenching. The quenched fluorescence was recovered with the addition of oxytetracycline due to the competition coordination [112]. This "turn-on" sensing strategy was expected to enhance the sensitivity because of a low background signal, and it was used for the detection of oxytetracycline hydrochloride with a LOD of $24.4 \mathrm{nM}$ in milk. However, it is likely that other ligands with a strong affinity for $\mathrm{Fe}^{3+}$ can also produce a similar signal increase.

In another example, a highly method was developed by using Au/Pt nanoclusters (NCs) capped with polyethyleneimine (Au/PtNCs@PEI) acting as a fluorescent nanoprobe. As shown in Fig. 9b2, the fluorescence was quenched upon addition of the target chlortetracycline, which was believed to aggregation the nanoprobes. The fluorescence of Au/PtNCs@PEI gradually weakened as the concentration of the target increased. A biosensor was developed for chlortetracycline detection in milk with a LOD of $0.35 \mu \mathrm{M}$ [113]. Similar quenching was also observed in the presence of tetracycline, oxytetracycline and doxycycline. Interestingly, when $\mathrm{Al}^{3+}$ was added, only the chlortetracycline sample showed a strong fluorescence recovery.

\section{Conclusions and future prospective}

In this review, we summarized various molecular recognition mechanisms coupled with nanomaterials for designing fluorescent biosensors to detect veterinary drug residues in foods. Many of these sensors were reported to meet the requirements of high sensitivity and accuracy, and sometimes high throughput detection and easy operation, which are critical for rapid, convenient, and quantitative detection. The detection strategies are very versatile, and can employ various target recognition elements (antibodies, aptamers, MIPs), assisting reagents (coated antigens, complementary DNA chains, SSB), signaling elements (different kinds of fluorescent nanomaterials or quenchers), and signal conversion methods (FRET, LRET, IFE, ET). Despite these progresses, this field still faces many challenges based on the contents reviewed. For example, nanomaterials are inherently large and heavy, leading to slow diffusion and thus a long detection time. In addition, nonspecific adsorption by nanomaterials could adversely affect detection. Many of the reviewed work required sophisticated surface functionalization of nanomaterials. Combined with variation in their synthesis, reproducibility could be an issue. High quality yet easy to use fluorescent nanomaterials are desirable for this purpose.

The fluorescence immunoassays so far relied on competitive approaches. The antibody-based "sandwich method" is not suitable for small-molecule detection due to steric hindrances. However, the strategy based on the small size splitting aptamers seems to be able to solve this problem. Currently, high-quality aptamers for veterinary drugs are still quite limited and most examples were demonstrated using the kanamycin aptamer. We expect more work to be done on the selection of aptamers for the drugs. In addition, we recommend rigorous testing of aptamer-based sensors using non-binding random DNA sequences to ensure the designed sensing mechanisms and avoiding potential artifacts [103].

So far, most of the detection required sample pretreatment and extraction of drug molecules into a solution phase. By introducing new nanomaterials (such as near-infrared and upconversion nanomaterials), it might be possible to achieve directly in situ detection, which can significantly improve food analysis. Taking advantage of the sharp emission 
peaks of many nanomaterials, in situ multiplexed detection would be even more desirable. Although some proof-of-concept work was performed with these materials, more rigorous and quantitative work on using these advanced optical materials and test the effect of various food matrix is needed.

The low matrix tolerance is a main bottleneck of the reported sensors leading to false-positive test results. For sample pretreatment, although immuno-affinity column are magnetic separation are commonly used to enrich the targets in the samples, these methods are quite expensive. MIPs appear to be a useful material for detection in food sample matrix, and this front can be further improved by combining MIP with aptamers [114]. With the rapid development of materials science and biotechnology, the development of novel, efficient and practical fluorescent biosensor systems based on nanomaterials are likely to be realized in the near future.

\section{Conflicts of interest}

None declared.

\section{Acknowledgments}

Funding from the Natural Sciences and Engineering Research Council of Canada (NSERC), and the National Natural Science Foundation of China (31672605) is acknowledged. X. Tao was supported by the China Scholarship Council (CSC) to visit the University of Waterloo.

\section{References}

[1] Manikandan VS, Adhikari B, Chen A. Nanomaterial based electrochemical sensors for the safety and quality control of food and beverages. Analyst 2018;143:4537-54.

[2] Li Z, Yu Y, Li Z, Wu T. A review of biosensing techniques for detection of trace carcinogen contamination in food products. Anal Bioanal Chem 2015;407:2711-26.

[3] Lee HC, Chen CM, Wei JT, Chiu HY. Analysis of veterinary drug residue monitoring results for commercial livestock products in Taiwan between 2011 and 2015. J Food Drug Anal 2018;26:565-71.

[4] Zhou JW, Zou XM, Song SH, Chen GH. Quantum dots applied to methodology on detection of pesticide and veterinary drug residues. J Agric Food Chem 2018;66:1307-19.

[5] Tian YF, Chen GH, Guo LH, Guo X, Mei XY. Methodology studies on detection of aminoglycoside residues. Food Anal Method 2015;8:1842-57.

[6] Dinos GP, Athanassopoulos CM, Missiri DA, Giannopoulou PC, Vlachogiannis IA, Papadopoulos GE, et al. Chloramphenicol derivatives as antibacterial and anticancer agents: historic problems and current solutions. Antibiotics 2016;5:20.

[7] Dorn JM, Alpern M, McNulty C, Volcheck GW. Sulfonamide drug allergy. Curr Allergy Asthma Rep 2018;18:38.

[8] Fellner MJ, Baer RL. Anaphylactic reaction to tetracycline in a penicillin-allergic patient: immunologic studies. JAMA 1965;192:997-8.
[9] $\mathrm{Wu} \mathrm{D}, \mathrm{Du} \mathrm{D}$, Lin Y. Recent progress on nanomaterial-based biosensors for veterinary drug residues in animal-derived food. TrAC Trends Anal Chem (Reference Ed) 2016;83: 95-101.

[10] Zhou D, Li Y, Huang L, Qian M, Li D, Sun G, et al. A reliable and cost-efficient TLC-HPLC method for determining total florfenicol residues in porcine edible tissues. Food Chem 2020;303:125399.

[11] Aman IM, Ahmed HF, Mostafa NY, Kitada Y, Kar G. Detection of tetracycline veterinary drug residues in Egyptian poultry meat by high performance liquid chromatography. J Vet Med Allied Sci 2017;1:52-8.

[12] Kawano S, Hao HY, Hashi Y, Lin JM. Analysis of chloramphenicol in honey by on-line pretreatment liquid chromatography-tandem mass spectrometry. Chin Chem Lett 2015;26:36-8.

[13] Shen J, Xia X, Jiang H, Li C, Li J, Li X, et al. Determination of chloramphenicol, thiamphenicol, florfenicol, and florfenicol amine in poultry and porcine muscle and liver by gas chromatography-negative chemical ionization mass spectrometry. J Chromatogr B: Anal Technol Biomed Life Sci 2009;877:1523-9.

[14] Moreno-Gonzalez D, Hamed AM, Gilbert-Lopez B, GamizGracia L, Garcia-Campana AM. Evaluation of a multiresidue capillary electrophoresis-quadrupole-time-of-flight mass spectrometry method for the determination of antibiotics in milk samples. J Chromatogr A 2017;1510:100-7.

[15] Flurer CL. Analysis of antibiotics by capillary electrophoresis. Electrophoresis 2003;24:4116-27.

[16] Lan L, Yao Y, Ping J, Ying Y. Recent advances in nanomaterial-based biosensors for antibiotics detection. Biosens Bioelectron 2017;91:504-14.

[17] Zhang X, Wu D, Zhou X, Yu Y, Liu J, Hu N, et al. Recent progress in the construction of nanozyme-based biosensors and their applications to food safety assay. TrAC Trends Anal Chem (Reference Ed) 2019;121:115668.

[18] Kumar H, Kuca K, Bhatia SK, Saini K, Kaushal A, Verma R, et al. Applications of nanotechnology in sensor-based detection of foodborne pathogens. Sensors 2020;20:1-19.

[19] Ramnani P, Saucedo NM, Mulchandani A. Carbon nanomaterial-based electrochemical biosensors for label-free sensing of environmental pollutants. Chemosphere 2016; 143:85-98.

[20] Xue Z, Zhang Y, Yu W, Zhang J, Wang J, Wan F, et al. Recent advances in aflatoxin $B 1$ detection based on nanotechnology and nanomaterials-A review. Anal Chim Acta 2019;1069:1-27.

[21] Kumar P, Kim KH, Deep A. Recent advancements in sensing techniques based on functional materials for organophosphate pesticides. Biosens Bioelectron 2015;70: 469-81.

[22] Sharma A, Khan R, Catanante G, Sherazi TA, Bhand S, Hayat A, et al. Designed strategies for fluorescence-based biosensors for the detection of mycotoxins. Toxins 2018;10: 197.

[23] Rhouati A, Hayat A, Mishra RK, Bueno D, Shahid SA, Muñoz R, et al. Ligand assisted stabilization of fluorescence nanoparticles; an insight on the fluorescence characteristics, dispersion stability and DNA loading efficiency of nanoparticles. J Fluoresc 2016;26:1407-14.

[24] Stolker AAM, Brinkman UAT. Analytical strategies for residue analysis of veterinary drugs and growth-promoting agents in food-producing animals-a review. J Chromatogr A 2005;1067:15-53.

[25] Białk-Bielińska A, Kumirska J, Stepnowski P. What do we know about the chronic and mixture toxicity of the residues of sulfonamides in the environment? Organic PollutantsMonitoring, Risk and Treatment. InTech; 2013. p. 59-86.

[26] Commission Regulation. Commission Regulation (EU) No 37/2010 of 22 December 2009 on pharmacologically active substances and their classification regarding maximum residue limits in foodstuffs of animal origin. Off $\mathrm{J}$ Eur Commun 2010:1-72. No. L15. 
[27] GB31650-2019. National food safety standard. Maximum residue limits for veterinary drugs in foods. Beijing, China: National health commission of the people's republic of China; 2019.

[28] Code of Federal Regulations. 556283. Washington, DC: U.S. Food and Drug Administration; 2012.

[29] System JPL. Available at: http://www.m5.ws001.squarestart. ne.jp/foundation/agrdtl.php; 2008.

[30] Beyene T. Veterinary drug residues in food-animal products: its risk factors and potential effects on public health. J Veterinar Sci Tech 2016;7:1000285.

[31] Kennedy DG, Cannavan A, McCracken RJ. Regulatory problems caused by contamination, a frequently overlooked cause of veterinary drug residues. J Chromatogr A 2000;882:37-52.

[32] Qiu S, Chen X, Chen P, Wang T, Hu S, Fan M, et al. Application of molecular imprinting technique in residue analysis of veterinary drugs in food. J Food Saf Qual 2015;6: 2248-55.

[33] Hames B, Ruiz R, Scarlata C, Sluiter A, Sluiter J, Templeton D. Preparation of samples for compositional analysis. In: Laboratory Analytical Procedure-Technical Report; 2008. NREL/TP-510-42620. Available at: http:// www.nrel.gov/biomass/analytical_procedures.html.

[34] Dongmei C, Yanfei T, Huan Y, Zonghui Y. Progress of sample pre-treatment in analysis of veterinary drug residues. Chem Bull 2009;8:713-9.

[35] Zhao JB, Zhao BJ, Qi HX, Zou QM, Chen Y, Zhang F, et al. Development of immunochromatographic strips based on covalently conjugated BHHCT-Eu ${ }^{3+} @ \mathrm{SiO}_{2}$ for rapid and quantitative detection of kanamycin. Chin J Anal Chem 2017;45:1467-74.

[36] Dehghani S, Danesh NM, Ramezani M, Alibolandi M, Lavaee P, Nejabat M, et al. A label-free fluorescent aptasensor for detection of kanamycin based on dsDNA-capped mesoporous silica nanoparticles and Rhodamine B. Anal Chim Acta 2018;1030:142-7.

[37] Zhang J, Shikha S, Mei Q, Liu J, Zhang Y. Fluorescent microbeads for point-of-care testing: a review. Microchim Acta 2019;186:361.

[38] Farias PM, Santos BS, Fontes A. Semiconductor fluorescent quantum dots: efficient biolabels in cancer diagnostics. Methods Mol Biol 2009:407-19.

[39] Peng T, Wang J, Zhao S, Zeng Y, Zheng P, Liang D, et al. Highly luminescent green-emitting Au nanocluster-based multiplex lateral flow immunoassay for ultrasensitive detection of clenbuterol and ractopamine. Anal Chim Acta 2018;1040:143-9.

[40] Panigrahi A, Sahu BP, Mandani S, Nayak D, Giri S, Sarma TK. AIE active fluorescent organic nanoaggregates for selective detection of phenolic-nitroaromatic explosives and cell imaging. J Photochem Photobiol, A 2019;374: 194-205.

[41] Wang Z, Hu S, Zhang G, Liu J, Xia J, Peng J, et al. Aggregation-induced emission-based competitive lateral flow immunoassay for rapid detection of sulfamethazine in honey. Food Agric Immunol 2019;30:1303-17.

[42] Zhang G, Xu S, Xiong Y, Duan H, Chen W, Li X, et al. Ultrabright fluorescent microsphere and its novel application for improving the sensitivity of immunochromatographic assay. Biosens Bioelectron 2019;135:173-80.

[43] Majdinasab M, Sheikh-Zeinoddin M, Soleimanian-Zad S, Li P, Zhang Q, Li X, et al. A reliable and sensitive timeresolved fluorescent immunochromatographic assay (TRFICA) for ochratoxin A in agro-products. Food Contr 2015;47:126-34.

[44] Zhang Z, Wang D, Li J, Zhang Q, Li P. Monoclonal antibody-europium conjugate-based lateral flow time-resolved fluoroimmunoassay for quantitative determination of T-2 toxin in cereals and feed. Anal Methods 2015;7:2822-9.

[45] Fang C, Wu S, Duan N, Dai S, Wang Z. Highly sensitive aptasensor for oxytetracycline based on upconversion and magnetic nanoparticles. Anal Methods 2015;7:2585-93.

[46] Ouyang Q, Liu Y, Chen Q, Guo Z, Zhao J, Li H, et al. Rapid and specific sensing of tetracycline in food using a novel upconversion aptasensor. Food Contr 2017;81:156-63.

[47] Ould-Ely T, Bawazer LA, Morse DE. Bio-inspired silica nanomaterials for biomedical applications. Compre Biomater II 2017;2:1-17.

[48] Ruggi A, Beekman C, Wasserberg D, Subramaniam V, Reinhoudt DN, van Leeuwen FW, et al. Dendritic ruthenium (II)-based dyes tuneable for diagnostic or therapeutic applications. Chem Eur 2011;17:464-7.

[49] Oroval M, Coll C, Bernardos A, Marcos MD, MartínezMáñez R, Shchukin DG, et al. Selective fluorogenic sensing of as (III) using aptamer-capped nanomaterials. ACS Appl Mater Interfaces 2017;9:11332-6.

[50] Kavruk M, Celikbicak O, Ozalp V, Borsa B, Hernandez F, Bayramoglu G, et al. Antibiotic loaded nanocapsules functionalized with aptamer gates for targeted destruction of pathogens. Chem Commun 2015;51:8492-5.

[51] Zhu K, Li J, Wang Z, Jiang H, Beier RC, Xu F, et al. Simultaneous detection of multiple chemical residues in milk using broad-specificity antibodies in a hybrid immunosorbent assay. Biosens Bioelectron 2011;26:2716-9.

[52] He Y, Wen X, Zhang B, Fan Z. Novel aptasensor for the ultrasensitive detection of kanamycin based on grapheneoxide quantum-dot-linked single-stranded DNA-binding protein. Sensor Actuator B 2018;265:20-6.

[53] Medintz IL, Uyeda HT, Goldman ER, Mattoussi H. Quantum dot bioconjugates for imaging, labelling and sensing. Nat Mater 2005;4:435-46.

[54] Wang Q, Chao Y. Multifunctional quantum dots and liposome complexes in drug delivery. J Biomed Res 2018;32: 91-106.

[55] Jiang W, Beloglazova NV, Wang Z, Jiang H, Wen K, de Saeger S, et al. Development of a multiplex flow-through immunoaffinity chromatography test for the on-site screening of 14 sulfonamide and 13 quinolone residues in milk. Biosens Bioelectron 2015;66:124-8.

[56] Zhu S, Meng Q, Wang L, Zhang J, Song Y, Jin H, et al. Highly photoluminescent carbon dots for multicolor patterning, sensors, and bioimaging. Angew Chem Int Ed 2013;52:3953-7.

[57] Liu ML, Chen BB, Li CM, Huang CZ. Carbon dots: synthesis, formation mechanism, fluorescence origin and sensing applications. Green Chem 2019;21:449-71.

[58] Xu D, Lin Q, Chang HT. Recent advances and sensing applications of carbon dots. Small Methods 2019;4:1900387.

[59] Goswami N, Lin F, Liu Y, Leong DT, Xie J. Highly luminescent thiolated gold nanoclusters impregnated in nanogel. Chem Mater 2016;28:4009-16.

[60] Zhang Y, Zhu C, Zhang L, Tan C, Yang J, Chen B, et al. DNA-templated silver nanoclusters for multiplexed fluorescent DNA detection. Small 2015;11:1385-9.

[61] Hosseini M, Mehrabi F, Ganjali MR, Norouzi P. A fluorescent aptasensor for sensitive analysis oxytetracycline based on silver nanoclusters. Luminescence 2016;31: 1339-43.

[62] Coons AH, Kaplan MH. Localization of antigen in tissue cells: II. Improvements in a method for the detection of antigen by means of fluorescent antibody. J Exp Med 1950; 91:1-13.

[63] Zhang W, He X, Liu P, Li W, Liu X. Rapid determination of ractopamine in porcine urine by a fluorescence immunochromatography assay. Anal Lett 2016;49:2165-76. 
[64] Ding S, Chen J, Jiang H, He J, Shi W, Zhao W, et al. Application of quantum dot-antibody conjugates for detection of sulfamethazine residue in chicken muscle tissue. J Agric Food Chem 2006;54:6139-42.

[65] Chen J, Xu F, Jiang H, Hou Y, Rao Q, Guo P, et al. A novel quantum dot-based fluoroimmunoassay method for detection of Enrofloxacin residue in chicken muscle tissue. Food Chem 2009;113:1197-201.

[66] Le T, Zhu L, Yang X. A quantum dot-based immunoassay for screening of tylosin and tilmicosin in edible animal tissues. Food Addit Contam 2015;32:719-24.

[67] Hu G, Sheng W, Zhang Y, Wu X, Wang S. A novel and sensitive fluorescence immunoassay for the detection of fluoroquinolones in animal-derived foods using upconversion nanoparticles as labels. Anal Bioanal Chem 2015;407: 8487-96.

[68] Hu G, Sheng W, Zhang Y, Wang J, Wu X, Wang S. Upconversion nanoparticles and monodispersed magnetic polystyrene microsphere based fluorescence immunoassay for the detection of sulfaquinoxaline in animal-derived foods. J Agric Food Chem 2016;64:3908-15.

[69] Sheng W, Li S, Liu Y, Wang J, Zhang Y, Wang S. Visual and rapid lateral flow immunochromatographic assay for enrofloxacin using dyed polymer microspheres and quantum dots. Microchim Acta 2017;184:4313-21.

[70] $\mathrm{Hu} \mathrm{LM}$, Luo K, Xia J, Xu GM, Wu CH, Han JJ, et al. Advantages of time-resolved fluorescent nanobeads compared with fluorescent submicrospheres, quantum dots, and colloidal gold as label in lateral flow assays for detection of ractopamine. Biosens Bioelectron 2017;91:95-103.

[71] Huang Z, Xiong Z, Chen Y, Hu S, Lai W. Sensitive and matrix-tolerant lateral flow immunoassay based on fluorescent magnetic nanobeads for the detection of clenbuterol in swine urine. J Agric Food Chem 2019;67:3028-36.

[72] Zhou J, Zhu K, Xu F, Wang W, Jiang H, Wang Z, et al. Development of a microsphere-based fluorescence immunochromatographic assay for monitoring lincomycin in milk, honey, beef, and swine urine. J Agric Food Chem 2014; 62:12061-6.

[73] Chen R, Li H, Zhang H, Zhang S, Shi W, Shen J, et al. Development of a lateral flow fluorescent microsphere immunoassay for the determination of sulfamethazine in milk. Anal Bioanal Chem 2013;405:6783-9.

[74] Wang Q, Pei X, Peng T, Xie J, Xie S, Sun Y, et al. Determination of tilmicosin by fluorescence-based immunochromatography. Anal Lett 2016;49:2052-62.

[75] Huang X, Aguilar ZP, Li H, Lai W, Wei H, Xu H, et al. Fluorescent $\mathrm{ru}(\mathrm{phen})_{3}^{2+}$-doped silica nanoparticles-based ICTS sensor for quantitative detection of enrofloxacin residues in chicken meat. Anal Chem 2013;85:5120-8.

[76] Xie Y, Zhang L, Yang X, Le T. Development of a quantum dot-based immunochromatography test strip for rapid screening of oxytetracycline and 4-epi-oxytetracycline in edible animal tissues. Food Addit Contam 2017;34:371-8.

[77] Beloglazova NV, Shmelin PS, Eremin SA. Sensitive immunochemical approaches for quantitative (FPIA) and qualitative (lateral flow tests) determination of gentamicin in milk. Talanta 2016;149:217-24.

[78] Shi CY, Deng N, Liang JJ, Zhou KN, Fu QQ, Tang Y. A fluorescent polymer dots positive readout fluorescent quenching lateral flow sensor for ractopamine rapid detection. Anal Chim Acta 2015;854:202-8.

[79] Sheng W, Chang Q, Shi Y, Duan W, Zhang Y, Wang S. Visual and fluorometric lateral flow immunoassay combined with a dual-functional test mode for rapid determination of tetracycline antibiotics. Microchim Acta 2018;185: 404

[80] Li X, Shen J, Wang Q, Gao S, Pei X, Jiang H, et al. Multiresidue fluorescent microspheres immunochromatographic assay for simultaneous determination of macrolides in raw milk. Anal Bioanal Chem 2015;407:9125-33.
[81] Zhao S, Wang S, Zhang S, Liu JH, Dong YY. State of the art: lateral flow assay (LFA) biosensor for on-site rapid detection. Chin Chem Lett 2018;29:1567-77.

[82] Zong C, Liu J. The arsenic binding aptamer cannot bind arsenic: critical evaluation of aptamer selection and binding. Anal Chem 2019;91:10887-93.

[83] Mehlhorn A, Rahimi P, Joseph Y. Aptamer-based biosensors for antibiotic detection: a review. Biosensors 2018;8:54.

[84] Song KM, Jeong E, Jeon W, Jo H, Ban C. A coordination polymer nanobelt (CPNB)-based aptasensor for sulfadimethoxine. Biosens Bioelectron 2012;33:113-9.

[85] Zhao H, Gao S, Liu M, Chang Y, Fan X, Quan X. Fluorescent assay for oxytetracycline based on a long-chain aptamer assembled onto reduced graphene oxide. Microchim Acta 2013;180:829-35.

[86] Ma X, Du C, Zhang J, Shang M, Song W. A system composed of vanadium(IV) disulfide quantum dots and molybdenum(IV) disulfide nanosheets for use in an aptamer-based fluorometric tetracycline assay. Mikrochim Acta 2019;186:837.

[87] Zhang L, Wang J, Deng J, Wang S. A novel fluorescent "turn-on" aptasensor based on nitrogen-doped graphene quantum dots and hexagonal cobalt oxyhydroxide nanoflakes to detect tetracycline. Anal Bioanal Chem 2020;412. 1343-51.

[88] Tan B, Zhao H, Du L, Gan X, Quan X. A versatile fluorescent biosensor based on target-responsive graphene oxide hydrogel for antibiotic detection. Biosens Bioelectron 2016; 83:267-73.

[89] Wang Y, Ma T, Ma S, Liu Y, Tian Y, Wang R, et al. Fluorometric determination of the antibiotic kanamycin by aptamer-induced FRET quenching and recovery between $\mathrm{MoS}_{2}$ nanosheets and carbon dots. Microchim Acta 2016; 184:203-10.

[90] Ha NR, Jung IP, La IJ, Jung HS, Yoon MY. Ultra-sensitive detection of kanamycin for food safety using a reduced graphene oxide-based fluorescent aptasensor. Sci Rep 2017; 7:40305.

[91] Sadeghi AS, Mohsenzadeh M, Abnous K, Taghdisi SM, Ramezani M. Development and characterization of DNA aptamers against florfenicol: fabrication of a sensitive fluorescent aptasensor for specific detection of florfenicol in milk. Talanta 2018;182:193-201.

[92] Esmaelpourfarkhani M, Abnous K, Taghdisi SM, Chamsaz M. A fluorometric assay for oxytetracycline based on the use of its europium(III) complex and aptamermodified silver nanoparticles. Microchim Acta 2019;186:290.

[93] Chen J, Li Z, Ge J, Yang R, Zhang L, Qu LB, et al. An aptamer-based signal-on bio-assay for sensitive and selective detection of kanamycin A by using gold nanoparticles. Talanta 2015;139:226-32.

[94] Dolati S, Ramezani M, Nabavinia MS, Soheili V, Abnous K, Taghdisi SM. Selection of specific aptamer against enrofloxacin and fabrication of graphene oxide based label-free fluorescent assay. Anal Biochem 2018;549:124-9.

[95] Yan Z, Yi H, Wang L, Zhou X, Yan R, Zhang D, et al. Fluorescent aptasensor for ofloxacin detection based on the aggregation of gold nanoparticles and its effect on quenching the fluorescence of Rhodamine B. Spectrochim Acta A Mol Biomol Spectrosc 2019;221:117203.

[96] Wang J, Lu T, Hu Y, Wang X, Wu Y. A label-free and carbon dots based fluorescent aptasensor for the detection of kanamycin in milk. Spectrochim Acta A Mol Biomol Spectrosc 2020;226:117651.

[97] Belal ASF, Ismail A, Elnaggar MM, Belal TS. Click chemistry inspired copper sulphide nanoparticle-based fluorescence assay of kanamycin using DNA aptamer. Spectrochim Acta A Mol Biomol Spectrosc 2018;205:48-54.

[98] Yuan F, Zhao H, Zhang Z, Gao L, Xu J, Quan X. Fluorescent biosensor for sensitive analysis of oxytetracycline based on an indirectly labelled long-chain aptamer. RSC Adv 2015;5: 58895-901. 
[99] Zhang H, Fang C, Wu S, Duan N, Wang Z. Upconversion luminescence resonance energy transfer-based aptasensor for the sensitive detection of oxytetracycline. Anal Biochem 2015;489:44-9.

[100] Ramezani M, Danesh NM, Lavaee P, Abnous K, Taghdisi SM. A selective and sensitive fluorescent aptasensor for detection of kanamycin based on catalytic recycling activity of exonuclease III and gold nanoparticles. Sens Actuators, B 2016;222:1-7.

[101] Zhang F, Wang S, Liu J. Gold nanoparticles adsorb DNA and aptamer probes too strongly and a comparison with graphene oxide for biosensing. Anal Chem 2019;91(22): 14743-50.

[102] Zong CH, Zhang ZJ, Liu BW, Liu JW. Adsorption of arsenite on gold nanoparticles studied with DNA oligonucleotide probes. Langmuir 2019;35:7304-11.

[103] Liu XX, Zhang F, Zhang Z, Huang Z, Liu J. Dopamine and melamine binding to gold nanoparticles dominates their aptamer-based label-free colorimetric sensing. Anal Chem 2020;92:9370-8.

[104] Wang CK, Chen D, Wang QQ, Tan R. Kanamycin detection based on the catalytic ability enhancement of gold nanoparticles. Biosens Bioelectron 2017;91:262-7.

[105] Feagin TA, Maganzini N, Soh HT. Strategies for creating structure-switching aptamers. ACS Sens 2018;3:1611-5.

[106] Song KM, Cho M, Jo H, Min K, Jeon SH, Kim T, et al. Gold nanoparticle-based colorimetric detection of kanamycin using a DNA aptamer. Anal Biochem 2011;415:175-81.

[107] Ahmad OS, Bedwell TS, Esen C, Garcia-Cruz A, Piletsky SA. Molecularly imprinted polymers in electrochemical and optical sensors. Trends Biotechnol 2019;37:294-309.

[108] Wang P, Fu XF, Li J, Luo J, Zhao XY, Sun MJ, et al. Preparation of hydrophilic molecularly imprinted polymers for tetracycline antibiotics recognition. Chin Chem Lett 2011;22: $611-4$.

[109] Hou J, Li H, Wang L, Zhang P, Zhou T, Ding H, et al. Rapid microwave-assisted synthesis of molecularly imprinted polymers on carbon quantum dots for fluorescent sensing of tetracycline in milk. Talanta 2016;146:34-40.

[110] Ton XA, Acha V, Haupt K, Bui B Tse Sum. Direct fluorimetric sensing of UV-excited analytes in biological and environmental samples using molecularly imprinted polymer nanoparticles and fluorescence polarization. Biosens Bioelectron 2012;36:22-8.

[111] Drakopoulos AI, Ioannou PC. Spectrofluorimetric study of the acid-base equilibria and complexation behavior of the fluoroquinolone antibiotics ofloxacin, norfloxacin, ciprofloxacin and pefloxacin in aqueous solution. Anal Chim Acta 1997;354:197-204.

[112] An X, Zhuo S, Zhang P, Zhu C. Carbon dots based turn-on fluorescent probes for oxytetracycline hydrochloride sensing. RSC Adv 2015;5:19853-8.

[113] Xu N, Meng L, Li HW, Lu DY, Wu Y. Polyethyleneimine capped bimetallic $\mathrm{Au} / \mathrm{Pt}$ nanoclusters are a viable fluorescent probe for specific recognition of chlortetracycline among other tetracycline antibiotics. Microchim Acta 2018; 185:294.

[114] Zhang Z, Liu J. Molecular imprinting with functional DNA. Small 2019;15:1805246. 